\title{
CONVERTIBLE BONDS IN A DEFAULTABLE DIFFUSION MODEL
}

\author{
Tomasz R. Bielecki* \\ Department of Applied Mathematics \\ Illinois Institute of Technology \\ Chicago, IL 60616, USA \\ Stéphane Crépey ${ }^{\dagger}$ \\ Département de Mathématiques \\ Université d'Évry Val d'Essonne \\ 91025 Évry Cedex, France \\ Monique Jeanblanc ${ }^{\ddagger}$ \\ Département de Mathématiques \\ Université d'Évry Val d'Essonne \\ 91025 Évry Cedex, France \\ and \\ Europlace Institute of Finance \\ Marek Rutkowski ${ }^{\S}$ \\ School of Mathematics \\ University of New South Wales \\ Sydney, NSW 2052, Australia \\ and \\ Faculty of Mathematics and Information Science \\ Warsaw University of Technology \\ 00-661 Warszawa, Poland
}

December 23, 2007

\footnotetext{
*The research of T.R. Bielecki was supported by NSF Grant 0202851 and Moody's Corporation grant 5-55411.

${ }^{\dagger}$ The research of S. Crépey was supported by Ito33.

¥The research of M. Jeanblanc was supported by Ito33 and Moody’s Corporation grant 5-55411.

$\S$ The research of M. Rutkowski was supported by the 2007 Faculty Research Grant PS12918.
} 


\section{Introduction}

In 4], working in an abstract set-up, we characterized arbitrage prices of generic Convertible Securities (CS), such as Convertible Bonds (CB), and we provided a rigorous decomposition of a CB into a bond component and a (game) option component, in order to give a definite meaning to commonly used terms of "CB spread" and "CB implied volatility." Moreover, in [5], we showed that in the hazard process set-up, the theoretical problem of pricing and hedging CS can essentially be reduced to a problem of solving a related doubly reflected Backward Stochastic Differential Equations (R2BSDE for short in the sequel, see [5]). Finally, in [6], we established the formal connection between this R2BSDE and related variational inequalities with double obstacle in a generic Markovian intensity model. The related mathematical issues are dealt with in Crépey [13, 14].

In this paper, we study CSs (in particular, CBs) in a specific market set-up. Namely, we consider a primary market consisting of: a savings account, a stock underlying a CS, and an associated credit default swap (CDS) (or, alternatively to the latter, a rolling $C D S$ more realistically used as an hedging instrument, see Section 2.2 and [7]). We model the dynamics of these three securities in terms of Markovian diffusion set-up with default (Section 2). In this model, we give conditions, obtained by applying the general results of [13, 14], which ensure that the R2BSDE related to a CS has a solution (Proposition 3.5), and we provide the associated (super-)hedging strategy (Theorem 3.2). Moreover, we characterize the pricing function of the CS in terms of viscosity solutions of associated variational inequalities (Theorem 3.3), and we prove the convergence of suitable approximation schemes (Theorem 3.4). We then specify these results to a convertible bond and its decomposition into straight bond and option components (Section 4).

The above-mentioned model appears as the simplest equity-to-credit reduced form model one may think of (the connection between equity and credit in the model being materialized by the fact that the default intensity $\gamma$ depends on the stock level $S$ ), and it is thus widely used in the industry for dealing with defaultable convertible bonds. This was the first motivation for the present study. The second motivation was the fact that all assumptions that we postulated in our previous theoretical works [4, 5, 6] are satisfied within this set-up; in this sense, the model is consistent with our theory of convertible securities. In particular, we worked in [4, 6] under the assumption that the value $U_{t}^{c b}$ of a CB upon a call at time $t$ yields, as a function of time, a well-defined process satisfying some natural conditions. In the specific framework of this paper, using uniqueness of arbitrage prices (Proposition 2.1 and Theorem 3.1) and a form of continuous aggregation property of the value $U_{t}^{c b}$ of a CB upon a call at time $t$ (Theorem 4.14(ii)), we are actually able to prove that this assumption is satisfied, and we also give ways to compute $U_{t}^{c b}$ (Theorems 4.14 and 4.15 .

\section{Model}

In this section, we introduce a simple specification of the generic Markovian default intensity set-up of [6. More precisely, we consider a defaultable diffusion model, with time and stock-dependent local default intensity and local volatility (see also [2, 1, 18, 19, 28, 11]).

\subsection{Canonical Construction}

Let us be given, relative to a finite horizon date $\bar{T}>0$, a filtered probability space $(\Omega, \mathbb{F}, \mathcal{G}, \mathbb{Q})$ satisfying the usual conditions, where $\mathbb{F}$ is the filtration of a standard Brownian motion $W$ on $[0, \bar{T}]$ under $\mathbb{Q}$. Here $\mathbb{Q}$ is devoted to represent a risk-neutral probability measure on a financial market to be defined below. We define the pre-default factor process $\widetilde{S}$ (to be interpreted later as the pre-default stock price of the firm underlying a $\mathrm{CB}$ ) as the following diffusion over $[0, \bar{T}]$ :

$$
d \widetilde{S}_{t}=\widetilde{S}_{t}\left(\left(r(t)-q(t)+\eta \gamma\left(t, \widetilde{S}_{t}\right)\right) d t+\sigma\left(t, \widetilde{S}_{t}\right) d W_{t}\right), \quad \widetilde{S}_{0}=x \in \mathbb{R}
$$


with related generator

$$
\mathcal{L} \equiv \partial_{t}+(r-q+\eta \gamma) S \partial_{S}+\frac{\sigma^{2} S^{2}}{2} \partial_{S^{2}}^{2}
$$

Assumption 2.1 (i) The riskless short interest rate $r(t)$, the equity dividend yield $q(t)$ and the local default intensity $\gamma(t, S) \geq 0$, are bounded Borel-measurable functions, and $\eta$ is a non-negative constant;

(ii) The local volatility $\sigma(t, S)$ is a positively bounded Borel-measurable function, so in particular $\sigma(t, S) \geq \underline{\sigma}$, for a positive constant $\underline{\sigma}$;

(iii) The functions $\gamma(t, S) S$ and $\sigma(t, S) S$ are Lipschitz continuous in $S$, uniformly in $t$.

Note that we authorize negative values of $r$ and/or $q$, in order, for instance, to possibly account for repo rates in the model.

Under these assumptions the SDE (1) admits a unique strong solution $\widetilde{S}$. Moreover, the following a priori estimate is available, for any $p \in[2,+\infty$ ) (see, e.g., [13]),

$$
\mathbb{E}_{\mathbb{Q}}\left[\sup _{t \in[0, \bar{T}]}\left|\widetilde{S}_{t}\right|^{p}\right] \leq C\left(1+|x|^{p}\right) .
$$

Remark 2.2 (i) For $x>0$ the solution of (1) is non-negative. In this work we find it convenient to define (1) for any initial condition $x \in \mathbb{R}$, even though only the positive values will have a financial interpretation. This will be useful for the variational inequalities approach (see Remark 3.10).

(ii) The fact that $\gamma$ may depend on $S$ in this model is crucial, since this dependence actually conveys all the 'equity-to-credit' information in the model. A natural choice for $\gamma$ is a decreasing (e.g., negative power) function of $\widetilde{S}$ capped when $\widetilde{S}$ is close to 0 . A possible refinement is to positively floor $\gamma$. The lower bound on $\gamma$ then represents pure credit risk, as opposed to equity-related credit risk.

We define the $[0, \bar{T}] \cup\{+\infty\}$-valued random default time $\tau_{d}$ by the so-called canonical construction [8]. Specifically, we set (by convention, $\inf \emptyset=\infty$ )

$$
\tau_{d}=\inf \left\{t \in[0, \bar{T}] ; \int_{0}^{t} \gamma\left(u, \widetilde{S}_{u}\right) d u \geq \varepsilon\right\}
$$

where $\varepsilon$ is a unit exponential random variable on $(\Omega, \mathbb{F}, \mathcal{G}, \mathbb{Q})$ independent of $W$. We set $H_{t}=\mathbb{1}_{\tau_{d} \leq t}$ and

$$
M_{t}^{d}=H_{t}-\int_{0}^{t}\left(1-H_{u}\right) \gamma\left(u, \widetilde{S}_{u}\right) d u .
$$

Let $\mathbb{H}$ be the filtration generated by the process $H$ and the filtration $\mathbb{G}$ be given as $\mathbb{F} \vee \mathbb{H}$. Because of our construction of $\tau_{d}$, the process $\gamma\left(t, \widetilde{S}_{t}\right)$ is the $\mathbb{F}$-intensity of $\tau_{d}$ and the process $M^{d}$ is a $\mathbb{G}$-martingale, called the compensated jump martingale. Moreover, the process

$$
\mathbb{P}\left(\tau>t \mid \mathcal{F}_{t}\right)=e^{-\int_{0}^{t} \gamma\left(u, \widetilde{S}_{u}\right) d u}
$$

is continuous and non-increasing. This implies in particular that the filtration $\mathbb{F}$ is immersed in $\mathbb{G}$ (Hypothesis $H$ holds, in the terminology of [8]), in the sense that all $\mathbb{F}$-martingales are $\mathbb{G}$-martingales. So the $\mathbb{F}$-Brownian motion $W$ is a $\mathbb{G}$-Brownian motion under $\mathbb{Q}$. Note also that $(\mathbb{F}, \mathbb{Q} ; W)$ has the local martingale predictable representation property, since we assumed that $\mathbb{F}$ is the filtration of the Brownian motion $W$ on $[0, \bar{T}]$.

\subsection{Specification of the Primary Market Model}

Given a reference entity (firm) with default time $\tau_{d}$ issuing a CS with maturity $T$, we consider on the time interval $[0, T]$ a primary market composed of the savings account and two primary risky 
assets:

- the stock $S$ of the reference entity, with fractional loss upon default assumed to be a constant $0 \leq \eta \leq 1$

- a CDS contract $B$ written at time 0 on the reference entity, with maturity $\bar{T}>T$, protection payment process given as a Borel-measurable bounded time-functional $\nu(t)$, and contracted $C D S$ spread $\bar{\nu}$.

We denote the discount factor process (inverse of the savings account) as $\beta$, so that $\beta_{t}=$ $e^{-\int_{0}^{t} r(u) d u}$. We also introduce the credit-risk adjusted discount factor $\alpha_{t}=e^{-\int_{0}^{t}\left(r(u)+\gamma\left(u, \widetilde{S}_{u}\right)\right) d u}$, where $\mu(t, S)=r(t)+\gamma(t, S)$.

Consistently with arbitrage requirements (cf. [6]), we assume that the dynamics of the pre-default price processes of the stock and of the CDS are given as $\widetilde{S}_{t}$ in 11 for $S$, and as $\widetilde{B}_{t}=\widetilde{B}\left(t, \widetilde{S}_{t}\right)$ for the CDS, where the (credit protection buyer) $C D S$ pre-default pricing function $\widetilde{B}(t, S)$ is the unique classical solution to the following PDE with generator $\mathcal{L}$ (cf. (2)):

$$
\mathcal{L} \widetilde{B}(t, S)+\delta(t, S)-\mu(t, S) \widetilde{B}(t, S)=0
$$

with terminal condition 0 at time $\bar{T}$, and where $\delta(t, S)=(\nu(t) \gamma(t, S)-\bar{\nu})$ is the pre-default dividend process of the CDS (as seen from the perspective of the protection buyer). We then define the stock and CDS price processes by, for $t \in[0, T]$ :

$$
S=H \widetilde{S}, B=H \widetilde{B}
$$

as well as the related discounted cumulative prices

$$
\widehat{S}_{t}=S_{t}+\beta_{t}^{-1} \int_{\left[0, t \wedge \tau_{d}\right]} \beta_{u} q(u) S_{u} d u, \widehat{B}_{t}=B_{t}+\beta_{t}^{-1} \int_{\left[0, t \wedge \tau_{d}\right]} \beta_{u}\left(\nu_{u} d H_{u}-\bar{\nu} d u\right)
$$

In the financial interpretation, $\widehat{S}$ and $\widehat{B}$ respectively denote the current value at time $t$ of a buy-and-hold strategy in one stock share and in one CDS contract at time 0, assuming that the related dividends are immediately reinvested in the savings account.

Since $\beta \widehat{S}$ and $\beta \widehat{B}$ are manifestly locally bounded processes, the arbitrage (risk-neutral) pricing measures on our primary market model are given by the probability measures $\widetilde{\mathbb{Q}} \sim \mathbb{Q}$ such that $\beta \widehat{S}$ and $\beta \widehat{B}$ are $(\mathbb{G}, \widehat{\mathbb{Q}})$-local martingale (see, e.g., $[\underline{6})$. Note that consistently with the general results of [5], we have for $t \in[0, T]$ :

$$
d\left(\begin{array}{c}
\beta_{t} \widehat{S}_{t} \\
\beta_{t} \widehat{B}_{t}
\end{array}\right)=\mathbb{1}_{\left\{t \leq \tau_{d}\right\}} \beta_{t} \Sigma_{t} d\left(\begin{array}{c}
W_{t} \\
M_{t}^{d}
\end{array}\right)
$$

where the $\mathbb{G}$-predictable dispersion matrix process $\Sigma$ is given by

$$
\Sigma_{t}=\left[\begin{array}{cc}
\sigma\left(t, \widetilde{S}_{t}\right) \widetilde{S}_{t} & -\eta \widetilde{S}_{t-} \\
\partial_{S} \widetilde{B}\left(t, \widetilde{S}_{t}\right) \sigma\left(t, \widetilde{S}_{t}\right) \widetilde{S}_{t} & \left(\nu(t)-\widetilde{B}_{t-}\right)
\end{array}\right] .
$$

So in particular $\mathbb{Q}$ is a risk-neutral measure on our primary market model. We work in the sequel under the following

Assumption $2.3 \Sigma$ is invertible on $\left[0, \tau_{d} \wedge T\right]$.

We then have the following

Proposition 2.1 For any risk-neutral probability measure $\widetilde{\mathbb{Q}}$ on the primary market, we have that $\mathbb{E}_{\mathbb{Q}}\left(\frac{d \widetilde{\mathbb{Q}}}{d \mathbb{Q}} \mid \mathcal{G}_{t}\right)=1$ on $\left[0, \tau_{d} \wedge T\right]$. 
Proof. Given a probability measure $\widetilde{\mathbb{Q}}$ equivalent to $\mathbb{Q}$ on $\left(\Omega, \mathcal{G}_{T}\right)$, the Radon-Nikodym density $Z_{t}=\mathbb{E}_{\mathbb{Q}}\left(\frac{d \widetilde{\mathbb{Q}}}{d \mathbb{Q}} \mid \mathcal{G}_{t}\right)$ is a positive $(\mathbb{G}, \mathbb{Q})$-martingale. Therefore, by Kusuoka [27], there exist two $\mathbb{G}$-predictable processes $\varphi$ and $\varphi^{d}$ such that

$$
d Z_{t}=Z_{t-}\left(\varphi_{t} d W_{t}+\varphi_{t}^{d} d M_{t}^{d}\right), \quad t \in[0, T]
$$

A probability measure $\widetilde{\mathbb{Q}}$ is then risk-neutral iff the process $\beta \widehat{X}$ is a $(\mathbb{G}, \widetilde{\mathbb{Q}})$-local martingale, or equivalently, if the processes $\beta \widehat{S} Z$ and $\beta \widehat{B} Z$ are $(\mathbb{G}, \mathbb{Q})$-local martingales. These conditions are satisfied if and only if

$$
\Sigma_{t}\left(\begin{array}{c}
\varphi_{t} \\
\gamma\left(t, \widetilde{S}_{t}\right) \varphi_{t}^{d}
\end{array}\right)=0
$$

Until $\tau_{d} \wedge T$ the unique solution to $(9)$ is $\varphi=\varphi^{d}=0$. We conclude that $Z=1$ on $\left[0, \tau_{d} \wedge T\right]$.

Proposition 2.1 shows that the postulated market model is complete, provided that all trading activities are stopped at the random time $\tau_{d} \wedge T$. It is also complete after default time $\tau_{d}$ if $\eta<1$, that is, the equity is still traded after default. It is worth stressing that the model is no longer complete after default if $\eta=1$, since in that case the only instrument traded after default is the savings account. In the latter case, the non-uniqueness of a risk-neutral probability measure holds.

\subsubsection{Rolling CDS}

In practice traders typically use a rolling $C D S$ (see [7]) as hedging instrument, rather than a plain CDS contract as considered above. The rolling CDS in fact corresponds to the wealth process of a self-financing trading strategy that amounts to continuously rolling one unit of long CDS contracts indexed by their inception date $t \in[0, T]$, with respective maturities $\bar{T}(t)$, where $\bar{T}$ is an increasing piecewise constant time-functional with $\bar{T}(T) \geq T$ (see [7] for details). We shall denote such contracts as $C D S(t, \bar{T}(t))$. This strategy amounts to holding at every time $t \in[0, T]$ one unit of the $C D S(t, \bar{T}(t))$. At time $t+d t$ the unit position in the $C D S(t, \bar{T}(t))$ is unwounded, the proceeds (which may be positive or negative depending on the evolution of the market between $t$ and $t+d t$ ) are reinvested in the savings account, and a freshly emitted $C D S(t+d t, \bar{T}(t+d t))$. is entered at no cost. This procedure is carried on in continuous time (to be understood as: every day, in a practical context of trading) until the hedging horizon $T$. In the case of a rolling CDS, the entry $\beta \widehat{B}$ in $(6)$ is then to be understood as the discounted cumulative value process of this strategy, and the only change wrt to the case of a standard CDS is that the dispersion matrix $\Sigma$ in (7) needs to be changed into (see Appendix A

$$
\Sigma_{t}=\left[\begin{array}{cc}
\sigma\left(t, \widetilde{S}_{t}\right) \widetilde{S}_{t} & -\eta \widetilde{S}_{t-} \\
\partial_{S} \widetilde{P}^{t}\left(t, \widetilde{S}_{t}\right) \sigma\left(t, \widetilde{S}_{t}\right) \widetilde{S}_{t}-\bar{\nu}\left(t, \widetilde{S}_{t}\right) \partial_{S} \widetilde{F}^{t}\left(t, \widetilde{S}_{t}\right) \sigma\left(t, \widetilde{S}_{t}\right) \widetilde{S}_{t} & \nu(t)
\end{array}\right]
$$

Here the functions $\widetilde{P}^{t}$ and $\widetilde{F}^{t}$ are the pre-default pricing functions of the protection leg and the fee leg, respectively, of $C D S(t, \bar{T}(t))$. They are characterized as the solutions of PDEs of the form (5) on $[t, \bar{T}(t)]$ with functions $\delta$ therein respectively given by $\delta^{1}(u, S)=\nu(u) \gamma(u, S)$ and $\delta^{2}(u, S)=1$, and where $\bar{\nu}\left(t, \widetilde{S}_{t}\right)=\frac{\widetilde{P}^{t}\left(t, \widetilde{S}_{t}\right)}{\widetilde{F}^{t}\left(t, \widetilde{S}_{t}\right)}$ denotes the related spread.

\section{Convertible Securities}

We now specify to the above model the notion of a convertible security, previously introduced in a general set-up in [4]. Let 0 (resp. $T$ with $0 \leq T \leq \bar{T}$ ) stand for the inception date (resp. the maturity date) of a convertible security (CS) with underlying $S$. For any $t \in[0, T]$, we write $\mathcal{F}_{T}^{t}$ (resp. $\mathcal{G}_{T}^{t}$ ) to denote the set of all $\mathbb{F}$-stopping times (resp. $\mathbb{G}$-stopping times) with values in $[t, T]$. 
Given the time of lifting of a call protection of a $C S, \bar{\tau} \in \mathcal{F}_{T}^{0}$, let also $\overline{\mathcal{F}}_{T}^{t}$ stand for $\left\{\varrho \in \mathcal{F}_{T}^{t} ; \varrho \geq \bar{\tau}\right\}$ and $\overline{\mathcal{G}}_{T}^{t}$ stand for $\left\{\varrho \in \mathcal{G}_{T}^{t} ; \varrho \wedge \tau_{d} \geq \bar{\tau} \wedge \tau_{d}\right\}$. Let finally $\tau$ denote $\tau_{p} \wedge \tau_{c}$ for any $\left(\tau_{p}, \tau_{c}\right) \in \mathcal{G}_{T}^{t} \times \overline{\mathcal{G}}_{T}^{t}$.

Definition 3.1 A Convertible Security with underlying $S$ is a game option (see [4, 5, 6, 26, 25]) with the ex-dividend cumulative discounted cash flows $\pi\left(t ; \tau_{p}, \tau_{c}\right)$ given by the formula, for any $t \in[0, T]$ and $\left(\tau_{p}, \tau_{c}\right) \in \mathcal{G}_{T}^{t} \times \overline{\mathcal{G}}_{T}^{t}$,

$$
\beta_{t} \pi\left(t ; \tau_{p}, \tau_{c}\right)=\int_{t}^{\tau} \beta_{u} d D_{u}+\mathbb{1}_{\left\{\tau_{d}>\tau\right\}} \beta_{\tau}\left(\mathbb{1}_{\left\{\tau=\tau_{p}<T\right\}} L_{\tau_{p}}+\mathbb{1}_{\left\{\tau_{c}<\tau_{p}\right\}} U_{\tau_{c}}+\mathbb{1}_{\{\tau=T\}} \xi\right),
$$

where:

- the dividend process $D=\left(D_{t}\right)_{t \in[0, T]}$ equals

$$
D_{t}=\int_{[0, t]}\left(1-H_{u}\right) d C_{u}+\int_{[0, t]} R_{u} d H_{u}
$$

for some coupon process $C=\left(C_{t}\right)_{t \in[0, T]}$, which is a $\mathbb{G}$-adapted càdlàg process with finite variation, and some real-valued, $\mathbb{G}$-predictable recovery process $R=\left(R_{t}\right)_{t \in[0, T]}$;

- the put payment $L$ is given as a $\mathbb{G}$-adapted, real-valued, càdlàg process on $[0, T]$,

- the call payment $U$ is a $\mathbb{G}$-adapted, real-valued, càdlàg process on $[0, T]$, such that

$$
L_{t} \leq U_{t} \text { for } t \in\left[\tau_{d} \wedge \bar{\tau}, \tau_{d} \wedge T\right) \text {, }
$$

- the payment at maturity $\xi$ is a $\mathcal{G}_{T}$-measurable real random variable.

In addition, the processes $R, L$ and the random variable $\xi$ are assumed to satisfy the following inequalities, for some positive constant $c$ :

$$
\begin{aligned}
& -c \leq R_{t} \leq c\left(1 \vee S_{t}\right), \quad t \in[0, T], \\
& -c \leq L_{t} \leq c\left(1 \vee S_{t}\right), \quad t \in[0, T], \\
& -c \leq \xi \leq c\left(1 \vee S_{T}\right) .
\end{aligned}
$$

Theorem 3.1 If the $\mathbb{Q}$-Dynkin game related to the CS admits a value $\Pi$, in the sense that

$$
\begin{aligned}
& \quad \operatorname{esssup}_{\tau_{p} \in \mathcal{G}_{T}^{t}} \operatorname{essinf}_{\tau_{c} \in \overline{\mathcal{G}}_{T}^{t}} \mathbb{E}_{\mathbb{Q}}\left(\pi\left(t ; \tau_{p}, \tau_{c}\right) \mid \mathcal{G}_{t}\right)=\Pi_{t} \\
& \quad=\operatorname{essinf}_{\tau_{c} \in \overline{\mathcal{G}}_{T}^{t}} \operatorname{esssup}_{\tau_{p} \in \mathcal{G}_{T}^{t}} \mathbb{E}_{\mathbb{Q}}\left(\pi\left(t ; \tau_{p}, \tau_{c}\right) \mid \mathcal{G}_{t}\right), \quad t \in[0, T],
\end{aligned}
$$

and $\Pi$ is a $\mathbb{G}$-semimartingale, then $\Pi$ is the unique arbitrage (ex-dividend) price of the CS.

Proof. Except for the uniqueness statement, this follows by applying the general results in [4. To verify the uniqueness property, we first note that given the estimate $(3)$ on $\widetilde{S}$ (hence $S$ ), the general results of 4 also imply that any arbitrage price of a CS is given by the value of the related Dynkin Game for some risk-neutral measure $\widetilde{\mathbb{Q}}$. Now, for any such risk-neutral measure $\widetilde{\mathbb{Q}}$, we have that $Z_{t}=\mathbb{E}_{\mathbb{Q}}\left(\frac{d \widetilde{\mathbb{Q}}}{d \mathbb{Q}} \mid \mathcal{G}_{t}\right)=1$ on $\left[0, \tau_{d} \wedge T\right]$, by Proposition 2.1 . Furthermore, $\pi\left(t ; \tau_{p}, \tau_{c}\right)$ is a $\mathcal{G}_{\tau_{d} \wedge T}$-measurable random variable. Therefore, for any $t \in[0, T], \tau_{p} \in \mathcal{G}_{T}^{t}, \tau_{c} \in \overline{\mathcal{G}}_{T}^{t}$,

$$
\mathbb{E}_{\widetilde{\mathbb{Q}}}\left(\pi\left(t ; \tau_{p}, \tau_{c}\right) \mid \mathcal{G}_{t}\right)=\mathbb{E}_{\mathbb{Q}}\left(\pi\left(t ; \tau_{p}, \tau_{c}\right) \mid \mathcal{G}_{t}\right) .
$$

In conclusion, the $\widetilde{\mathbb{Q}}$-Dynkin Game also has value $\Pi$.

We now define special cases of CSs, corresponding to American-style and European-style CSs, respectively. Formally,

Definition 3.2 A non-callable CS (denoted as PB, cf. 4]) is a convertible security with $\bar{\tau}=T$, or, equivalently, $\bar{U}=\infty$. An Elementary Security (ES) is a non-callable CS with bounded variation dividend process $D$ over $[0, T]$, bounded payment at maturity $\xi$, and such that

$$
\int_{[0, t]} \beta_{u} d D_{u}+\mathbb{1}_{\left\{\tau_{d}>t\right\}} \beta_{t} L_{t} \leq \int_{[0, T]} \beta_{u} d D_{u}+\mathbb{1}_{\left\{\tau_{d}>T\right\}} \beta_{T} \xi, \quad t \in[0, T) .
$$


By Definition 3.2, PBs and ESs are special cases of CSs. Note that, given Theorem 3.1, a PB (resp. an ES) can be redefined in a more standard way as a financial product with ex-dividend cumulative discounted cash flows $\bar{\pi}\left(t ; \tau_{p}\right)$ (resp. $\phi(t)$ ) given as, for $t \in[0, T]$ and $\tau_{p} \in \mathcal{G}_{T}^{t}$,

$$
\beta_{t} \bar{\pi}\left(t ; \tau_{p}\right)=\int_{t}^{\tau_{p}} \beta_{u} d D_{u}+\mathbb{1}_{\left\{\tau_{d}>\tau_{p}\right\}} \beta_{\tau_{p}}\left(\mathbb{1}_{\left\{\tau_{p}<T\right\}} L_{\tau_{p}}+\mathbb{1}_{\left\{\tau_{p}=T\right\}} \xi\right)
$$

(resp. $\beta_{t} \phi(t)=\int_{t}^{T} \beta_{u} d D_{u}+\mathbb{1}_{\left\{\tau_{d}>T\right\}} \beta_{T} \xi$ for every $\left.t \in[0, T]\right)$.

Returning to the case of a general CS, we further postulate in the Markovian set-up of this paper, that

Assumption 3.3 - the coupon process $C_{t}=C(t):=\int_{[0, t]} c(u) d u+\sum_{0 \leq T_{i} \leq t} c^{i}$, for a bounded Borel-measurable continuous-time coupon rate function $c(\cdot)$ and deterministic discrete times and coupons $T_{i}$ and $c^{i}$, respectively; for reasons that will become clear in Section 4.7 , we take the tenor of the discrete coupons as $T_{0}=0<T_{1}<\cdots<T_{K-1}<T_{K}$, with $T \leq T_{K}$;

- the recovery process $R_{t}$ is of the form $R\left(t, S_{t-}\right)$ for a Borel-measurable function $R$;

- $\xi=\xi\left(S_{T}\right), L_{t}=L\left(t, S_{t}\right), U_{t}=U\left(t, S_{t}\right)$ for a Borel-measurable function $\xi$ and Borel-measurable functions $L, U$ such that for any $t, S$, we have

$$
L(t, S) \leq U(t, S), \quad L(T, S) \leq \xi(S) \leq U(T, S) .
$$

Definition 3.4 We define the accrued interest at time $t$ by

$$
A_{t}=\frac{t-T_{i_{t}-1}}{T_{i_{t}}-T_{i_{t}-1}} c^{i_{t}}
$$

where $i_{t}$ is the integer satisfying $T_{i_{t}-1} \leq t<T_{i_{t}}$ and we let $\rho(t)=\frac{c^{i_{t}}}{T_{i_{t}}-T_{i_{t}-1}}$. Note that on open intervals between the discrete coupon dates we have $d A_{t}=\rho(t) d t$. We also set

$$
\gamma_{t}=\gamma\left(t, \widetilde{S}_{t}\right), \quad \mu(t, S)=r(t)+\gamma(t, S), \quad \mu_{t}=\mu\left(t, \widetilde{S}_{t}\right),
$$

so that $\alpha_{t}=e^{-\int_{0}^{t} \mu_{u} d u}$ and for $t \in[0, T]$ (with the convention that $A_{0-}=0$ )

$$
\alpha_{t} A_{t}=\int_{[0, t]} d(\alpha A)_{u}=\int_{0}^{t} \alpha_{u}\left(\rho_{u}-\mu_{u} A_{u}\right) d u-\sum_{0 \leq T_{i} \leq t} \alpha_{T_{i}} c^{i} .
$$

To a CS with data (functions) $C, R, \xi, L, U$ and lifting time of call protection $\bar{\tau} \in \mathcal{F}_{T}^{0}$, we associate the Borel-measurable functions $f(t, S, \theta)$ (for $\theta$ real), $g(S), \ell(t, S)$ and $h(t, S)$ defined by

$$
g(S)=\xi(S)-A_{T}, \quad \ell(t, S)=L(t, S)-A_{t}, \quad h(t, S)=U(t, S)-A_{t},
$$

and

$$
f(t, S, \theta)=\gamma(t, S) R(t, S)+\Gamma(t, S)-\mu(t, S) \theta,
$$

where in turn $\Gamma(t, S)=c(t)+\rho(t)-\mu(t, S) A_{t}$. In the case of a non-callable CS, the process $U$ is irrelevant, and thus we may and do set $h(t, S)=+\infty$. Moreover, we note that in the case of an ES, which is a special case of non-callable CS, the process $L$ plays no role and thus we set $\ell(t, S)=-\infty$. Finally, we define the processes and random variables associated to a CS (parameterized by $\theta \in \mathbb{R}$, regarding $f$ ) as

$$
f_{t}(\theta)=f\left(t, \widetilde{S}_{t}, \theta\right), \quad g=g\left(\widetilde{S}_{T}\right), \quad \ell_{t}=\ell\left(t, \widetilde{S}_{t}\right), \quad h_{t}=h\left(t, \widetilde{S}_{t}\right)
$$

In order to ensure stability of solutions to the related BSDEs (see below) and, incidentally, to ensure well definedness of the previous processes, we work henceforth under the following

Assumption 3.5 The functions $r, \gamma, g, \ell, h, R, c$ are continuous. 


\subsection{Doubly Reflected BSDEs Approach}

We define:

$\mathcal{H}^{2}$ - the set of real-valued, $\mathbb{F}$-predictable processes $\Theta$ such that $\mathbb{E}_{\mathbb{Q}}\left[\int_{0}^{T} \Theta_{t}^{2} d t\right]<\infty$, $\mathcal{S}^{2}$ - the set of real-valued, $\mathbb{F}$-adapted, continuous processes $\Theta$ such that

$$
\mathbb{E}_{\mathbb{Q}}\left[\sup _{t \in[0, T]} \Theta_{t}^{2}\right]<\infty
$$

$\mathcal{A}^{2}$ - the space of finite variation continuous processes $K$ with (continuous and non decreasing) Jordan components $K^{ \pm} \in \mathcal{S}^{2}$ null at time 0 , $\mathcal{A}_{i}^{2}$ - the space of non-decreasing processes in $\mathcal{A}^{2}$.

For any $K \in \mathcal{A}^{2}$, we thus have that $K=K^{+}-K^{-}$, where $K^{ \pm} \in \mathcal{A}_{i}^{2}$ define mutually singular measures on $\mathbb{R}^{+}$.

Given a CS with data $C, R, \xi, L, U, \bar{\tau}$ and the associated processes and random variables $\left(f_{t}, g, \ell_{t}, h_{t}\right)$, we introduce the following doubly reflected Backward Stochastic Differential Equation $(\mathcal{E})$ with data $\left(f_{t}, g, \ell_{t}, h_{t}, \bar{\tau}\right)(\mathrm{R} 2 \mathrm{BSDE}$ for short, see [6, 13]), such that for $t \in[0, T]$ :

$$
\left.\begin{array}{c}
-d \Theta_{t}=f_{t}\left(\Theta_{t}\right) d t+d K_{t}-Z_{t} d W_{t}, \quad \Theta_{T}=g, \\
\ell_{t} \leq \Theta_{t} \leq \bar{h}_{t}, \\
\left(\Theta_{t}-\ell_{t}\right) d K_{t}^{+}=\left(\bar{h}_{t}-\Theta_{t}\right) d K_{t}^{-}=0,
\end{array}\right\}(\mathcal{E})
$$

where we set $\bar{h}_{t}=\mathbb{1}_{\{t<\bar{\tau}\}} \infty+\mathbb{1}_{\{t \geq \bar{\tau}\}} h_{t}$, using the convention that $0 \times \pm \infty=0$.

Definition 3.6 (i) By a solution to $(\mathcal{E})$, we mean a triple of processes

$$
(\Theta, Z, K) \in \mathcal{S}^{2} \times \mathcal{H}^{2} \times \mathcal{A}^{2}
$$

that satisfies all conditions in $(\mathcal{E})$ for any $0 \leq t \leq T$. In particular, $\Theta$ and $K$ have to be continuous processes.

(ii) In the case where $\bar{\tau}=T$, we have $K^{-}=0$, so that $(\mathcal{E})$ reduces to a reflected BSDE with data $(f, g, \ell)$ and $K \in \mathcal{A}_{i}^{2}$ in the solution.

(iii) In the special case of an ES, one can show that $K=0$ in any solution $(\Theta, Z, K)$ to $(\mathcal{E})$, so that $(\mathcal{E})$ reduces to an elementary $\operatorname{BSDE}$ with data $(f, g)$ and no process $K$ involved in the solution, referred to as $\left(\mathcal{E}^{\prime}\right)$ in what follows.

The following definition is standard, accounting for the dividends on the primary market.

Definition 3.7 By a (self-financing) primary strategy, we mean a pair $\left(V_{0}, \zeta\right)$ such that:

- $V_{0}$ is a $\mathcal{G}_{0}$-measurable real-valued random variable representing the initial wealth,

- $\zeta$ is an $\mathbb{R}^{1 \otimes 2}$-valued (bi-dimensional row vector), $\beta \widehat{X}$-integrable process representing holdings (number of units held) in primary risky assets,

- The wealth process $V$ of a primary strategy $\left(V_{0}, \zeta, Q\right)$ is given by

$$
d\left(\beta_{t} V_{t}\right)=\zeta_{t} d\left(\beta_{t} \widehat{X}_{t}\right), \quad t \in[0, T]
$$

with the initial condition $V_{0}$.

In the set-up of this paper, the notions of issuer (super)hedge and holder (super)hedge introduced in [6, 5] take the following form. Recall that we denote $\tau=\tau_{p} \wedge \tau_{c}$. 
Definition 3.8 Given a CS with ex-dividend cumulative discounted cash flows $\pi\left(t ; \tau_{p}, \tau_{c}\right)$ (cf. (11)):

(i) An issuer hedge for the game option is represented by a triplet $\left(V_{0}, \zeta, \tau_{c}\right)$ such that:

- $\left(V_{0}, \zeta\right)$ is a primary strategy with the wealth process $V$,

- $\tau_{c}$ belongs to $\overline{\mathcal{G}}_{T}^{0}$,

- the following inequality is valid, for every put time $\tau_{p} \in \mathcal{G}_{T}^{0}$,

$$
\beta_{\tau} V_{\tau} \geq \beta_{0} \pi\left(0 ; \tau_{p}, \tau_{c}\right), \quad \text { a.s. }
$$

(ii) A holder hedge for the game option is a quadruplet $\left(V_{0}, \zeta, \tau_{p}\right)$ such that:

- $\left(V_{0}, \zeta\right)$ is a primary strategy with the wealth process $V$,

- $\tau_{p}$ belongs to $\mathcal{G}_{T}^{0}$,

- the following inequality is valid, for every call time $\tau_{c} \in \overline{\mathcal{G}}_{T}^{0}$,

$$
\beta_{\tau} V_{\tau} \geq-\beta_{0} \pi\left(0 ; \tau_{p}, \tau_{c}\right), \quad \text { a.s. }
$$

By applying general results of [5, 6], we obtain the following (super-)hedging theorem.

Theorem 3.2 Let $(\widehat{\Theta}, Z, K)$ be a solution to $(\mathcal{E})$, assumed to exist, and let $\Theta_{t}$ denote $\mathbb{1}_{\left\{t<\tau_{d}\right\}} \widetilde{\Theta}_{t}$ with $\widetilde{\Theta}:=\widehat{\Theta}+A$. Then $\Theta$ is the unique arbitrage price process of the $C S$, and for any $t \in[0, T]$ :

(i) An issuer hedge with initial wealth $\Theta_{t}$ is furnished by

$$
\tau_{c}^{*}=\inf \left\{u \in[\bar{\tau} \vee t, T] ; \widehat{\Theta}_{u}=h_{u}\right\} \wedge T \in \overline{\mathcal{F}}_{T}^{t},
$$

and

$$
\zeta_{u}^{*}:=\mathbb{1}_{u \leq \tau_{d}}\left[Z_{u}, R_{u}-\widetilde{\Theta}_{u-}\right] \Sigma_{u}^{-1}, \quad t \leq u \leq T .
$$

Moreover, the corresponding wealth process is bounded from below and $\Theta_{t}$ is the smallest initial wealth of an issuer hedge.

(ii) A holder hedge with initial wealth $-\Theta_{t}$ is furnished by

$$
\tau_{p}^{*}=\inf \left\{u \in[t, T] ; \widehat{\Theta}_{u}=\ell_{u}\right\} \wedge T \in \mathcal{F}_{T}^{t}
$$

and $\zeta=-\zeta^{*}$ above. Moreover, $-\Theta_{t}$ is the smallest initial wealth of a holder hedge.

Proof. Applying the general results of [6], we see that $\Theta$ satisfies all the assumptions for $\Pi$ in Theorem 3.1. therefore, it is the unique arbitrage price process of the CS. Moreover, under Assumption 2.3 . (i) and (ii) follow by an application of the general results of [5, 6].

We thus see that in the present set-up any CS has a bilateral hedging price (bilateral in the sense that this price $\Theta_{t}$ ensures super-hedging to both the issuer and the holder of the claim, starting from the initial wealth $\Theta_{t}$ for the former and $-\Theta_{t}$ for the latter), which is also the unique arbitrage price. Of course, this conclusion hinges on our temporary assumption that the related BSDE has a solution.

\subsection{Variational Inequalities Approach}

Let $\mathcal{D}$ denote a closed sub-domain of $[0, T] \times \mathbb{R}$ given by either $[0, T] \times \mathbb{R}$ itself, or $[0, T] \times(-\infty, \bar{S}]$ for some $\bar{S}<\infty$. Let then

$$
\operatorname{Int}_{p} \mathcal{D}=[0, T) \times \mathbb{R} \text { or }[0, T) \times(-\infty, \bar{S}), \quad \partial_{p} \mathcal{D}=\mathcal{D} \backslash \operatorname{Int}_{p} \mathcal{D}
$$

stand for the parabolic interior and the parabolic boundary of $\mathcal{D}$, respectively. Let $\mathcal{P}$ be the class of functions $\Theta$ on $\mathcal{D}$ bounded by $C\left(1+|S|^{p}\right.$ ) for some real $C$ and integer $p$ (that may depend on $\Theta$ )

In order to establish the connection between the previous BSDEs and the formally related obstacles problems (see 6, 13, 14), we postulate henceforth the following

\footnotetext{
${ }^{1}$ By a slight abuse of terminology, we shall say that a function $\Theta(S, .$.$) is of class \mathcal{P}$ if it has polynomial growth in $S$, uniformly in any other arguments.
} 
Assumption $3.9 r, q, \gamma$ and $\sigma$ are continuous functions and the functions $(R, g, h, \ell)$ associated to a CS are (continuous and) of class $\mathcal{P}$ (or $h=+\infty$ in the case where $\bar{\tau}=T$, and $\ell=-\infty$ in the case of an ES).

Given a continuous boundary condition $b$, where $b$ is a continuous function of class $\mathcal{P}$ on $\partial_{p} \mathcal{D}$ such that $b=g$ pointwise at $T$, we introduce the following obstacles problem $(\mathcal{V I})$ on $\mathcal{D}$ (where $\mathcal{L}$ and $f$ were defined in $(18)$ and $(2)$, respectively)

$$
\max (\min (-\mathcal{L} \Theta(t, S)-f(t, S, \Theta(t, S)), \Theta(t, S)-\ell(t, S)), \Theta(t, S)-h(t, S))=0,
$$

supplemented by the boundary condition $\Theta=b$ on $\partial_{p} \mathcal{D}$.

Remark 3.10 Note that $(\mathcal{V I})$ is defined over a domain in space variable $S$ going to $-\infty$, though only the positive part of the domain has a financial interpretation (cf. Remark 2.2(i)). If we decided to pose problems $(\mathcal{V I})$ over bounded spatial domains, then we would need to impose some appropriate non-trivial boundary condition at the lower space boundary, in order to get a well-posed problem.

We refer the reader to Appendix $B$ for the definition of viscosity solutions which is relevant to cope with the time-discontinuities of $f$ at the $T_{i}$ s (assuming that the product under consideration pays discrete coupons). Building upon Definition B.1, we introduce the following definition of $\mathcal{P}$ $($ semi-)solutions to $(\mathcal{V I})$ on $\mathcal{D}$.

Definition 3.11 By a $\mathcal{P}$-subsolution, resp. supersolution, resp.resp. solution $\Theta$ of $(\mathcal{V I})$ on $\mathcal{D}$ for the boundary condition $b$, we mean a viscosity subsolution, resp. supersolution, resp.resp. solution of $(\mathcal{V} \mathcal{I})$ of class $\mathcal{P}$ on $\operatorname{Int}_{p} \mathcal{D}$, such that $\Theta \leq b$, resp. $\Theta \geq b$, resp.resp. $\Theta=b$, pointwise on $\partial_{p} \mathcal{D}$.

Theorem 3.3 Let $(\Theta, Z, K)$ be a solution to $(\mathcal{E})$, assumed to exist. Then:

(a) Cauchy problem: $\bar{\tau}=\mathbf{0}$. The process $\Theta$, denoted here as $\widehat{\Theta}$, can be written as $\widehat{\Theta}_{t}=\widehat{\Theta}\left(t, \widetilde{S}_{t}\right)$, where the function $\widehat{\Theta}$ is a $\mathcal{P}$-solution of $(\mathcal{V I})$ on $[0, T] \times \mathbb{R}$ with terminal condition $g$ at $T$;

(b) Cauchy-Dirichlet problem: $\bar{\tau}=\inf \left\{\mathbf{t}>\mathbf{0} ; \widetilde{\mathbf{S}}_{\mathbf{t}} \geq \overline{\mathbf{S}}\right\} \wedge \mathbf{T}$ for some $\overline{\mathbf{S}}>\mathbf{0}$. The process $\Theta$, denoted here as $\bar{\Theta}$, can be written on $[0, \bar{\tau}]$ as $\bar{\Theta}\left(t, \widetilde{S}_{t}\right)$, where the function $\bar{\Theta}$ is a $\mathcal{P}$-solution of $(\mathcal{V I})$ on $[0, T] \times(-\infty, \bar{S}]$ with terminal condition $g$ at $T$ and Dirichlet condition $\widehat{\Theta}(\cdot, \bar{S})$ at level $\bar{S}$ (where $\widehat{\Theta}$ is the function defined in (a)).

Proof. This follows by the application of the general results of Crépey [14, 13]. Note, in particular, that $\bar{\tau}$ depends a.s.-continuously on the initial condition $(t, x)$ of $\widetilde{S}$, under Assumption 2.1 (ii) (see, for instance, Darling and Pardoux [17]), which is one of the conditions postulated for (b) in [13] .

We now come to the issues of uniqueness and approximation of solutions for $(\mathcal{V I})$. For this, we make the following additional

Assumption 3.12 The functions $r, q, \gamma, \sigma$ are locally Lipschitz continuous.

We refer the reader to Crépey [14] or Barles and Souganidis [3] for the definition of stable, monotone and consistent approximation schemes to $(\mathcal{V I})$ and for the related notion of convergence of the scheme, involved in the following

Theorem 3.4 Let $(\Theta, z, K)$ be a solution to $(\mathcal{E})$, assumed to exist, and let the functions $\widehat{\Theta}$ and $\bar{\Theta}$ be defined as in Theorem 3.3 .

(a) Cauchy problem: $\bar{\tau}=\mathbf{0}$. The function $\widehat{\Theta}$ is the unique $\mathcal{P}$-solution, the maximal $\mathcal{P}$-subsolution, and the minimal $\mathcal{P}$-supersolution, of $(\mathcal{V I})$ on $\mathcal{D}=[0, T] \times \mathbb{R}$ with terminal condition $g$ at $T$. Let 
$\left(\Theta_{h}\right)_{h>0}$ denote a stable, monotone and consistent approximation scheme for the function $\widehat{\Theta}$. Then $\Theta_{h} \rightarrow \widehat{\Theta}$ locally uniformly on $\mathcal{D}$ as $h \rightarrow 0^{+}$.

(b) Cauchy-Dirichlet problem: $\bar{\tau}=\inf \left\{\mathbf{t}>\mathbf{0} ; \widetilde{\mathbf{S}}_{\mathbf{t}} \geq \overline{\mathbf{S}}\right\} \wedge \mathbf{T}$ for some $\overline{\mathbf{S}}>\mathbf{0}$. The function $\bar{\Theta}$ is the unique $\mathcal{P}$-solution, the maximal $\mathcal{P}$-subsolution, and the minimal $\mathcal{P}$-supersolution, of $(\mathcal{V I})$ on $\mathcal{D}=[0, T] \times(-\infty, \bar{S}]$ with terminal condition $g$ at $T$ and Dirichlet condition $\widehat{\Theta}(\cdot, \bar{S})$ at $\bar{S}$. Let $\left(\Theta_{h}\right)_{h>0}$ denote a stable, monotone and consistent approximation scheme for the function $\bar{\Theta}$. Then $\Theta_{h} \rightarrow \bar{\Theta}$ locally uniformly on $\mathcal{D}$ as $h \rightarrow 0^{+}$, provided $\Theta_{h} \rightarrow \bar{\Theta}(=\widehat{\Theta})$ at $\bar{S}$.

Proof. Note, in particular, that under our assumptions:

- the functions $(r(t)-q(t)+\eta \gamma(t, S)) S$ and $\sigma(t, S) S$ are locally Lipschitz continuous;

- the function $f$ admits a modulus of continuity in $S$, in the sense that for every $R>0$ there exists a nonnegative function $\eta_{R}$ continuous and null at 0 such that:

$$
\left|f(t, S, \theta)-f\left(t, S^{\prime}, \theta\right)\right| \leq \eta_{R}\left(\left|S-S^{\prime}\right|\right)
$$

for any $t \in[0, T]$ and $S, S^{\prime}, \theta \in \mathbb{R}$ with $|S|,\left|S^{\prime}\right|,|\theta| \leq R$.

The results then follows by an application of the general results of Crépey [14.

The previous results show the importance of having a solution $(\Theta, z, K)$ to $(\mathcal{E})$. By application of the general results of [13], we have the following

Proposition 3.5 Assume further that $\ell(t, S)=\lambda(t, S) \vee c$ for a function $\lambda$ of class $\mathcal{C}^{1,2}$ with

$$
\lambda, \partial_{t} \lambda, S \partial_{S} \lambda, S^{2} \partial_{S^{2}}^{2} \lambda \text { of class } \mathcal{P}
$$

and for a constant $c \in \mathbb{R} \cup\{-\infty\}$. Then $(\mathcal{E})$ admits a unique solution $(\Theta, z, K)$.

Example 3.13 The standing example for the function $\lambda(t, S)$ in Proposition 3.5 is $\lambda(t, S)=S$. In that case, $\ell$ corresponds to the payoff function of a call option (or, more precisely, to the lower payoff function of a convertible bond, see Section 4 .

Remark 3.14 We refer, in particular, the reader to the last section of Crépey [14] regarding the fact that the potential discontinuities of $f$ at the $T_{i}$ s (which represent a non-standard feature from the point of view of the classic theory of viscosity solutions as presented, for instance, in Crandall et al. [12]) are not a real issue in the previous results, provided one works with the suitable Definition B.1 of viscosity solutions to our problems.

\section{Application to Convertible Bonds}

\subsection{Convertible Bonds and Reduced Convertible Bonds}

As we already pointed out, a convertible bond is a special case of a convertible security. To describe the covenants of a typical convertible bond (CB), we need to introduce the following additional notation (see [4] for a thorough description and discussion of the convertible bonds covenants):

$\bar{N}$ : the par (nominal) value,

$\eta$ : the fractional loss on the underlying equity upon default $(0 \leq \eta \leq 1)$,

$\bar{R}_{t}$ : the recovery process on the CB upon default of the issuer at time $t$, given by $\bar{R}_{t}=\bar{R}\left(t, S_{t-}\right)$ for a continuous bounded function $\bar{R}$,

$\kappa:$ the conversion factor,

$R_{t}^{c b}=R^{c b}\left(t, S_{t-}\right)=(1-\eta) \kappa S_{t-} \vee \bar{R}_{t}:$ the effective recovery process,

$\xi^{c b}=\bar{N} \vee \kappa S_{T}+A_{T}$ : the effective payoff at maturity, 
$\bar{P} \leq \bar{C}$ : the put and call nominal payments, respectively, such that $\bar{P} \leq \bar{N} \leq \bar{C}$,

$\delta \geq 0$ : the length of the call notice period (see below),

$t^{\delta}=(t+\delta) \wedge T:$ the end date of the call notice period started at $t$.

Real-life convertible bonds typically include a positive call notice period $\delta$ so that if the issuer calls the bond at time $\tau_{c}$, then the holder may either redeem the bond for $\bar{C}$ or convert the bond into $\kappa$ shares of stock, at any time $u$ in $\left[\tau_{c}, \tau_{c}^{\delta}\right]$, where $\tau_{c}^{\delta}=\left(\tau_{c}+\delta\right) \wedge T$. Accounting for accrued interest, the effective call/conversion payment to the holder at time $u$ is $\bar{C} \vee \kappa S_{u}+A_{u}$.

This clause makes $\mathrm{CB}$ with positive call notice period difficult to price directly. To handle this, we developed in [4] a two step approach to value a $\mathrm{CB}$ with positive call notice period. In the first step, we value the $\mathrm{CB}$ upon call as a Reduced Convertible Bond (RB, see Definition 4.1 below). In the second step, we use this price as the payoff at call time of a $\mathrm{CB}$ with no call notice period.

Definition 4.1 (4]) A reduced convertible bond (RB) is a convertible security with recovery process $R^{c b}$ and terminal payoffs $L^{c b}, U^{c b}, \xi^{c b}$ such that

$$
R_{\tau_{d}}^{c b}=(1-\eta) \kappa S_{\tau_{d}-} \vee \bar{R}_{\tau_{d}}, \quad L_{t}^{c b}=\bar{P} \vee \kappa S_{t}+A_{t}, \quad \xi^{c b}=\bar{N} \vee \kappa S_{T}+A_{T},
$$

and

$$
U_{t}^{c b}=\mathbb{1}_{\left\{t<\tau_{d}\right\}} \widetilde{U}^{c b}\left(t, S_{t}\right)+\mathbb{1}_{\left\{t \geq \tau_{d}\right\}}\left(\bar{C} \vee \kappa S_{t}+A_{t}\right), \quad t \in[0, T],
$$

for a function $\widetilde{U}^{c b}(t, S)$ jointly continuous in time and space, except for negative left jumps of $-c^{i}$ at the $T_{i}$ s, and such that $\widetilde{U}^{c b}\left(t, S_{t}\right) \geq \bar{C} \vee \kappa S_{t}+A_{t}$ on the event $\left\{t<\tau_{d}\right\}$ (so $U_{t}^{c b} \geq \bar{C} \vee \kappa S_{t}+A_{t}$ for every $t \in[0, T])$.

So, the discounted dividend process of an $\mathrm{RB}$ is given by, for every $t \in[0, T]$,

$$
\int_{[0, t]} \beta_{u} d D_{u}^{c b}:=\int_{\left[0, t \wedge \tau_{d}\right]} \beta_{u} c(u) d u+\sum_{0 \leq T_{i} \leq t, T_{i}<\tau_{d}} \beta_{T_{i}} c^{i}+\mathbb{1}_{\left\{0 \leq \tau_{d} \leq t\right\}} \beta_{\tau_{d}} R_{\tau_{d}}^{c b}
$$

Clearly, a CB with no notice period $(\delta=0)$ is an $\mathrm{RB}$, with

$$
\widetilde{U}^{c b}(t, S)=\bar{C} \vee \kappa S+A_{t} .
$$

More generally, the financial interpretation of the process $U^{c b}$ in an $\mathrm{RB}$ is that $U_{t}^{c b}$ represents the value of the $\mathrm{RB}$ upon a call at time $t$. In Section 4.7, we shall prove that under mild regularity assumptions in our model, any CB (whether $\delta$ is positive or not) can be interpreted and priced as an RB.

\subsection{Decomposition of a Reduced Convertible Bond}

\subsubsection{Embedded Bond}

We consider an RB with dividend process $D^{c b}$ given by 25 , and an ES with the same coupon process as the RB and with $R^{b}$ and $\xi^{b}$ as follows:

$$
R_{t}^{b}=\bar{R}_{t}, \quad \xi^{b}=\bar{N}+A_{T},
$$

so that

$$
R_{t}^{c b}-R_{t}^{b}=\left((1-\eta) \kappa S_{t}-\bar{R}_{t}\right)^{+} \geq 0, \quad \xi^{c b}-\xi^{b}=\left(\kappa S_{T}-\bar{N}\right)^{+} \geq 0 .
$$

Thus, this ES corresponds to the defaultable bond with discounted cash flows given by the expression

$$
\begin{aligned}
\beta_{t} \phi_{t} & =\int_{t}^{T} \beta_{u} d D_{u}^{b}+\mathbb{1}_{\left\{\tau_{d}>T\right\}} \beta_{T} \xi^{b} \\
& :=\int_{t}^{T \wedge \tau_{d}} \beta_{u} c(u) d u+\sum_{t<T_{i} \leq T, T_{i}<\tau_{d}} \beta_{T_{i}} c^{i}+\mathbb{1}_{\left\{t<\tau_{d} \leq T\right\}} \beta_{\tau_{d}} R_{\tau_{d}}^{b}+\mathbb{1}_{\left\{\tau_{d}>T\right\}} \beta_{T} \xi^{b}
\end{aligned}
$$


and the associated functions

$$
f(t, S, \theta)=\gamma(t, S) R^{b}(t, S)+\Gamma(t, S)-\mu(t, S) \theta, \quad g(S)=\bar{N} .
$$

This bond can be seen as the pure bond component of the RB (that is, the RB stripped of its optional clauses). Therefore, we shall call it the bond embedded into the $R B$, or simply the embedded bond.

In the sequel, in addition to the assumptions made so far, we work under the following reinforcement of Assumption 3.12 .

Assumption 4.2 The functions $r(t), q(t), \gamma(t, S) S, \sigma(t, S) S, \gamma(t, S) \bar{R}(t, S)$ and $c(t)$ are continuously differentiable in time, and thrice continuously differentiable in space, with bounded related spatial partial derivatives.

Note that these assumptions cover typical financial applications. In particular, they are satisfied when $\bar{R}$ is constant and for well-chosen parameterizations of $\sigma$ and $\gamma$, which can be enforced at the time of the calibration of the model.

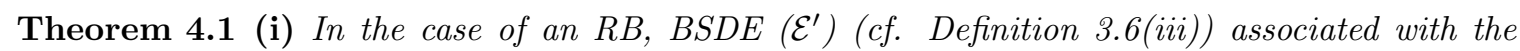
embedded bond admits a solution $(\widehat{\Phi}, Z)$. Denoting $\widetilde{\Phi}=\widehat{\Phi}+A$, the embedded bond admits a unique arbitrage price

$$
\Phi_{t}=\mathbb{1}_{t<\tau_{d}} \widetilde{\Phi}_{t}, \quad t \in[0, T] .
$$

(ii) Moreover we have $\widehat{\Phi}_{t}=\widehat{\Phi}\left(t, \widetilde{S}_{t}\right)$, where the function $\widehat{\Phi}(t, S)$ is bounded, jointly continuous in time and space and twice continuously differentiable in space, and the process $\widehat{\Phi}\left(t, \widetilde{S}_{t}\right)$ is an Ito process with true martingale component, such that

$$
\begin{aligned}
d \widehat{\Phi}_{t} & =u_{t} d t+v_{t} d W_{t} \\
& :=\left(\mu_{t} \widehat{\Phi}_{t}-\left(\gamma_{t} R_{t}^{b}+\Gamma_{t}\right)\right) d t+\sigma\left(t, \widetilde{S}_{t}\right) \widetilde{S}_{t} \partial_{S} \widehat{\Phi}_{t} d W_{t}
\end{aligned}
$$

with $v \in \mathcal{H}^{2}$.

Proof. (i) By standard results (see, e.g., 20, 22]), BSDE $\left(\mathcal{E}^{\prime}\right)$ with data $\left(\gamma R^{b}+\Gamma-\mu \Theta, \bar{N}\right)$ admits a solution $(\widehat{\Phi}, Z)$. Hence, by Theorem 3.2 (specified to the particular case of an ES), we obtain that the embedded bond admits a unique arbitrage price given by 27).

(ii) By $\left(\mathcal{E}^{\prime}\right)$, we have

$$
\widehat{\Phi}_{t}=\mathbb{E}_{\mathbb{Q}}\left(\int_{t}^{T}\left(\gamma_{u} R_{u}^{b}+\Gamma_{u}-\mu_{u} \widehat{\Phi}_{u}\right) d u+\left(\xi^{b}-A_{T}\right) \mid \mathcal{F}_{t}\right), \quad t \in[0, T],
$$

or equivalently (see [6]),

$$
\alpha_{t} \widehat{\Phi}_{t}=\mathbb{E}_{\mathbb{Q}}\left(\int_{t}^{T} \alpha_{u}\left(\gamma_{u} R_{u}^{b}+\Gamma_{u}\right) d u+\alpha_{T}\left(\xi^{b}-A_{T}\right) \mid \mathcal{F}_{t}\right), \quad t \in[0, T],
$$

and thus, using (17),

$$
\alpha_{t} \widetilde{\Phi}_{t}=\mathbb{E}_{\mathbb{Q}}\left(\int_{t}^{T} \alpha_{u}\left(\gamma_{u} R_{u}^{b} d u+c(u)\right) d u+\sum_{t<T_{i} \leq T} \alpha_{T_{i}} c^{i}+\alpha_{T} \xi^{b} \mid \mathcal{F}_{t}\right), \quad t \in[0, T] .
$$

Set

$$
\begin{aligned}
\alpha_{t} \widehat{\Phi}_{t}^{0} & =\mathbb{E}_{\mathbb{Q}}\left(\int_{t}^{T} \alpha_{u}\left(\gamma_{u} R_{u}^{b}+c(u)\right) d u+\alpha_{T}\left(\bar{N}+A_{T}\right) \mid \mathcal{F}_{t}\right), \quad t \leq T, \\
\alpha_{t} \widehat{\Phi}_{t}^{i} & =\mathbb{E}_{\mathbb{Q}}\left(\alpha_{T_{i}} c^{i} \mid \mathcal{F}_{t}\right), \quad t \leq T_{i} .
\end{aligned}
$$


We have $\widetilde{\Phi}_{T}=\widehat{\Phi}_{T}^{0}$ and $\widetilde{\Phi}_{t}=\widehat{\Phi}_{t}^{0}+\sum_{j ; T_{i} \leq T_{j} \leq T} \widehat{\Phi}_{t}^{j}$ on $\left[T_{i-1}, T_{i}\right)$, or on $\left[T_{K-1}, T\right)$ in case $i=K-1$. Let us denote generically $T$ or $T^{i}$ by $\mathcal{T}$, and $\widehat{\Phi}^{0}$ or $\widehat{\Phi}^{i}$ by $\Theta$, as appropriate according to the problem at hand. Note that $\Theta$ is bounded. Moreover, given our regularity assumptions, we have $\Theta_{t}=\widehat{\Theta}\left(t, \widetilde{S}_{t}\right)$, where $\widehat{\Theta}$ belongs to $\mathcal{C}^{1,2}([0, \mathcal{T}) \times \mathbb{R}) \cap \mathcal{C}^{0}([0, \mathcal{T}] \times \mathbb{R})$ (see [34, 22]). Therefore, $\widehat{\Phi}_{t}=\widetilde{\Phi}_{t}-A_{t}$ is given by $\widehat{\Phi}\left(t, \widetilde{S}_{t}\right)$ for a function $\widehat{\Phi}(t, S)$, which is jointly continuous in time and space on $[0, T] \times \mathbb{R}$ and twice continuously differentiable in space on $[0, T) \times \mathbb{R}$. Moreover, given (29), (30) and the above $\mathcal{C}^{1,2}$ regularity results, we have

$$
\begin{gathered}
d \widehat{\Phi}_{t}^{0}=\left(\mu_{t} \widehat{\Phi}_{t}^{0}-\left(\gamma_{t} R_{t}^{b}+c(t)\right)\right) d t+\sigma\left(t, \widetilde{S}_{t}\right) \widetilde{S}_{t} \partial_{S} \widehat{\Phi}^{0}\left(t, \widetilde{S}_{t}\right) d W_{t}, \quad t<T, \\
d \widehat{\Phi}_{t}^{i}=\mu_{t} \widehat{\Phi}_{t}^{i} d t+\sigma\left(t, \widetilde{S}_{t}\right) \widetilde{S}_{t} \partial_{S} \widehat{\Phi}^{i}\left(t, \widetilde{S}_{t}\right) d W_{t}, \quad i=1,2, \ldots, K, t<T_{i} \wedge T, \\
d A_{t}=\rho_{t} d t, \quad t \neq T_{i}, \quad i=0,1, \ldots, K .
\end{gathered}
$$

This yields

$$
d \widehat{\Phi}\left(t, \widetilde{S}_{t}\right)=\left(\mu_{t} \widetilde{\Phi}_{t}-\left(\gamma_{t} R_{t}^{b}+c(t)+\rho\right)\right) d t+\sigma\left(t, \widetilde{S}_{t}\right) \widetilde{S}_{t} \partial_{S} \widetilde{\Phi}\left(t, \widetilde{S}_{t}\right) d W_{t}=u_{t} d t+v_{t} d W_{t} .
$$

Moreover, since $\widehat{\Phi}$ and $u$ in 28 are bounded, we conclude that $v \in \mathcal{H}^{2}$.

\subsubsection{Embedded Game Exchange Option}

We now define the embedded Game Exchange Option as an RB with the dividend process $D^{c b}-D^{b}$, payment at maturity $\xi^{c b}-\xi^{b}$, put payment $L_{t}^{c b}-\Phi_{t}$, call payment $U_{t}^{c b}-\Phi_{t}$ and call protection lifting time $\bar{\tau}$. This means that the embedded Game Exchange Option is a zero-coupon CS with cash flows

$$
\begin{aligned}
& \beta_{t} \psi\left(t ; \tau_{p}, \tau_{c}\right)=\mathbb{1}_{\left\{t<\tau_{d} \leq \tau\right\}} \beta_{\tau_{d}}\left(R_{\tau_{d}}^{c b}-R_{\tau_{d}}^{b}\right) \\
& \quad+\mathbb{1}_{\left\{\tau_{d}>\tau\right\}} \beta_{\tau}\left(\mathbb{1}_{\left\{\tau=\tau_{p}<T\right\}}\left(L_{\tau_{p}}^{c b}-\Phi_{\tau_{p}}\right)+\mathbb{1}_{\left\{\tau=\tau_{c}<\tau_{p}\right\}}\left(U_{\tau_{c}}^{c b}-\Phi_{\tau_{c}}\right)+\mathbb{1}_{\{\tau=T\}}\left(\xi^{c b}-\xi^{b}\right)\right) .
\end{aligned}
$$

Note that from the point of view of the financial interpretation (see [4 for more about this), the Game Exchange Option corresponds to an option to exchange the embedded bond for either $L^{c b}, U^{c b}$ or $\xi^{c b}$ (as seen from the perspective of the holder), according to which player decides first to stop this game (and before $T$ or not).

We have the following observation.

Proposition 4.2 Given an $R B$, the associated functions $f(t, S, \theta), g=g(S), \ell=\ell(t, S)$ and $h=$ $h(t, S)$ are:

- $f=\gamma R^{c b}+\Gamma-\mu \theta, g=\bar{N} \vee \kappa S, \ell=\bar{P} \vee \kappa S, h=\widetilde{U}^{c b}-A$, for the $R B$;

- $f=\gamma\left(R^{c b}-R^{b}\right)-\mu \theta, g=(\kappa S-\bar{N})^{+}, \ell=\bar{P} \vee \kappa S-\widehat{\Phi}, h=\widetilde{U}^{c b}-A-\widehat{\Phi}$, for the embedded Game Exchange Option.

\subsection{Solution of the Doubly Reflected BSDEs}

Theorem 4.3 (i) The functions $f, g, \ell, h$ associated to an $R B$ or to the embedded Game Exchange Option (cf. Proposition 4.2), satisfy all the general assumptions of Theorems 3.33 .4$.

(ii) The related problems $(\mathcal{E})$ have unique solutions.

Proof. (i) The result for the $\mathrm{RB}$ follows directly from the definition of $\mathrm{RB}$. Then, in view of Proposition 4.2 the result for the Game Exchange Option follows from Theorem 4.1(ii).

(ii) In the case of the RB, we are in the situation of Example 3.13 , so the related problem $(\mathcal{E})$ has a unique solution $(\widehat{\Pi}, V, K)$, by an application of Proposition 3.5. Now, $(\widehat{\Phi}, Z)$ denoting the solution 
to the BSDE $\left(\mathcal{E}^{\prime}\right)$ exhibited in Theorem 4.1(i), it is immediate to check that $(\widehat{\Psi}, W, K)$ solves the Game Exchange Option-related problem $(\mathcal{E})$ iff $(\widehat{\Phi}+\widehat{\Psi}, Z+W, K)$ solves the RB-related problem $(\mathcal{E})$, whence in turn the result for the Game Exchange Option.

Given an RB, we denote by $\widehat{\Pi}$ and $\widehat{\Psi}$ the state-processes (first components $\Theta$ ) of solutions to the related R2BSDEs.

Theorem 4.4 (i) The process $\Psi_{t}$ defined as $\mathbb{1}_{t<\tau_{d}} \widehat{\Psi}_{t}$ is the unique arbitrage price of the embedded Game Exchange Optionand $\left(\Psi_{t}, \zeta^{*}, \tau_{c}^{*}\right)$ (resp. $\left(-\Psi_{t},-\zeta^{*}, \tau_{p}^{*}\right)$ ) as defined in Theorem 3.2 is an issuer hedge with initial value $\Psi_{t}$ (resp. holder hedge with initial value $-\Psi_{t}$ ) starting from time $t$ for the embedded Game Exchange Option;

(ii) The process $\Pi_{t}$ defined as $\mathbb{1}_{t<\tau_{d}} \widetilde{\Pi}_{t}$, with $\widetilde{\Pi}:=\widehat{\Pi}+A$, is the unique arbitrage price of the $R B$, and $\left(\Pi_{t}, \zeta^{*}, \tau_{c}^{*}\right)$ (resp. $\left.\left(-\Pi_{t},-\zeta^{*}, \tau_{p}^{*}\right)\right)$ as defined in Theorem 3.2 is an issuer hedge with initial value $\Pi_{t}$ (resp. holder hedge with initial value $-\Pi_{t}$ ) starting from time $t$ for the $R B$.

(iii) With $\widehat{\Phi}$ and $\Phi$ defined as in Theorem 4.1. we have $\Pi=\Phi+\Psi, \widehat{\Pi}=\widehat{\Phi}+\widehat{\Psi}$.

Proof. Given Theorem 4.3(i), statements (i) and (ii) follow by an application of Theorem 3.2 , whereas (iii) then follows from the general results in 4 .

In the foregoing sub-sections, we will give analytical characterizations of the so-called pre-default clean prices (pre-default pricwe shall accrued interest; see [6]) in terms of viscosity solutions to the associated variational inequalities. To get the corresponding pre-default prices, it suffices to add to the clean price process the related accrued interest process. Note that in the case of the Game Exchange Option, there are no discrete coupons involved and thus the pre-default clean price and the pre-default price coincide.

\subsection{Variational Inequalities for the No-Protection Clean Prices}

We first assume that $\bar{\tau}=0$ (no call protection). By application of Theorems 4.3, 3.3(a) and 3.4(a), we obtain the following result.

Theorem 4.5 (No-Protection Clean Prices) In the case where $\bar{\tau}=0$ (no call protection), we define the following problems on $\mathcal{D}=[0, T] \times \mathbb{R}$ :

(a) Defaultable Bond

$$
\begin{gathered}
-\mathcal{L} \widehat{\Phi}+\mu \widehat{\Phi}-\left(\gamma R^{b}+\Gamma\right)=0, \quad t<T, \\
\widehat{\Phi}(T, S)=\bar{N},
\end{gathered}
$$

(b) Game Exchange Option

$$
\begin{gathered}
\max \left(\min \left(-\mathcal{L} \widehat{\Psi}+\mu \widehat{\Psi}-\gamma\left(R^{c b}-R^{b}\right), \widehat{\Psi}-(\bar{P} \vee \kappa S-\widehat{\Phi})\right), \widehat{\Psi}-\left(\widetilde{U}^{c b}-A-\widehat{\Phi}\right)\right)=0, \quad t<T, \\
\widehat{\Psi}(T, S)=(\kappa S-\bar{N})^{+},
\end{gathered}
$$

(c) $R B$

$$
\begin{gathered}
\max \left(\min \left(-\mathcal{L} \widehat{\Pi}+\mu \widehat{\Pi}-\left(\gamma R^{c b}+\Gamma\right), \widehat{\Pi}-\bar{P} \vee \kappa S\right), \widehat{\Pi}-\left(\widetilde{U}^{c b}-A\right)\right)=0, \quad t<T, \\
\widehat{\Pi}(T, S)=\bar{N} \vee \kappa S .
\end{gathered}
$$

Then for any of the problems above, there exists a $\mathcal{P}$-solution on $\mathcal{D}$, denoted generically as $\widehat{\Theta}(t, S)$, that determines the corresponding No Protection (Pre-default) Clean Price, say $\widehat{\Theta}_{t}$, in the sense that

$$
\widehat{\Theta}_{t}=\widehat{\Theta}\left(t, \widetilde{S}_{t}\right), \quad t \in[0, T] .
$$

Moreover, we have uniqueness of the $\mathcal{P}$-solution and any stable, monotone and consistent approximation scheme for $\widehat{\Theta}$ converges locally uniformly to $\widehat{\Theta}$ on $\mathcal{D}$ as $h \rightarrow 0^{+}$. 
Corollary 4.6 A pair of No Protection Pre-default optimal stopping times $\left(\tau_{p}^{*}, \tau_{c}^{*}\right)$ (see Theorem 3.2), both in the case of the Game Exchange Option embedded in the RB and of the RB itself, is given by

$$
\begin{aligned}
\tau_{p}^{*} & =\inf \left\{u \in[t, T] ; \widetilde{S}_{u} \in \mathcal{E}_{p}\right\} \wedge T, \\
\tau_{c}^{*} & =\inf \left\{u \in[t, T] ; \widetilde{S}_{u} \in \mathcal{E}_{c}\right\} \wedge T,
\end{aligned}
$$

where

$$
\begin{gathered}
\mathcal{E}_{p}:=\{(t, S) \in[0, T] ; \widehat{\Pi}(t, S)=\bar{P} \vee \kappa S\}, \\
\mathcal{E}_{c}:=\left\{(t, S) \in[0, T] ; \widehat{\Pi}(t, S)=\widetilde{U}^{c b}(t, S)-A_{t}\right\},
\end{gathered}
$$

are the No Protection Pre-default Put or Conversion Region and the No Protection Pre-default Call Region.

Proof. This follows immediately of Theorems 4.5 and 4.4

Assuming that there is no call protection and that the RB is still alive at time $t$ :

- an optimal call time for the issuer of the RB is given by the first hitting time of $\mathcal{E}_{c}$ by $\widetilde{S}$ after $t$, if any such hitting time occurs before $T \wedge \tau_{d}$;

- an optimal put/conversion time for the holder of the RB consists in putting or converting, whichever is best, at the first hitting time of $\mathcal{E}_{p}$ by $\widetilde{S}$ after $t$, if any such hitting time occurs before $T \wedge \tau_{d}$

\subsection{Variational Inequalities for the Post-protection Prices}

For any $\bar{\tau} \in \mathcal{F}_{T}^{0}$, the associated Pre-default Price coincides on $[\bar{\tau}, T]$ with the Pre-default Price corresponding to a lifting time of call protection that would be given by $\bar{\tau}^{0}:=0$. This follows from the general results in [5], using also the fact that the BSDEs related to the problems with lifting times of call protection $\bar{\tau}$ and $\bar{\tau}^{0}$ both have solutions, by the previous results.

Thus No Protection Prices (pre-default prices for lifting time of call protection $:=\bar{\tau}^{0}=0$ ) can be also be interpreted as Post-protection (Pre-default) Prices for arbitrary $\bar{\tau} \in \mathcal{G}_{T}^{0}$. Therefore, the results of Section 4.4 also apply to Post-Protection Clean Prices. We thus obtain the following result.

Theorem 4.7 (Post-protection Clean Prices) Let $\bar{\tau} \in \mathcal{F}_{T}^{0}$. Then for an $R B$, the embedded Bond and the embedded Game Exchange Option, the corresponding Post-Protection Pre-default Clean Price process coincides on $[\bar{\tau}, T]$ with process $\widehat{\Theta}\left(t, \widetilde{S}_{t}\right)$, where $\widehat{\Theta}$ is the related function in Theorem 4.5.

Corollary 4.8 The pair of No-Protection Pre-default optimal stopping times $\left(\tau_{p}^{*}, \tau_{c}^{*}\right)$, and the associated No-Protection Pre-default Call and Put Regions $\mathcal{E}_{c}$ and $\mathcal{E}_{p}$ (see Corollary 4.6), can also be interpreted as a pair of Post-protection optimal stopping times and Post-protection Call and Put Regions, respectively.

So, assuming that call protection have already been lifted (namely, for $t \geq \bar{\tau}$ ) and that the RB is still alive, we conclude that:

- an optimal call time for the issuer of the RB is given by the first hitting time of $\mathcal{E}_{c}$ by $\widetilde{S}$ after $t$, if any such hitting time occurs before $T \wedge \tau_{d}$;

- an optimal put/conversion time for the holder of the RB consists in putting or converting, whichever is best, at the first hitting time of $\mathcal{E}_{p}$ by $\widetilde{S}$ after $t$, if any such hitting time occurs before $T \wedge \tau_{d}$. 


\subsection{Variational Inequalities for the Protection Prices}

We finally consider Protection Clean Prices $\bar{\Theta}$, namely, by definition, Pre-Default Clean Prices stopped at $\bar{\tau}$. As usual, to get the corresponding Protection (Pre-default) Prices, it suffices to add the related accrued interest process (if there are any discrete coupons involved). Let $\widehat{\Phi}, \widehat{\Psi}$ and $\widehat{\Pi}$ denote the No Protection Clean Prices Functions defined in Theorem 4.5 ,

\subsubsection{Hard Call Protections}

In the case of hard call protection $\bar{\tau}=\bar{T}$ for some $\bar{T} \leq T$, the protection clean prices functions $\bar{\Theta}$ are solutions of analytical problems as in Theorem 4.5(a) (special case with one obstacle, $h=+\infty$ ) with $\bar{T}$ instead of $T$, and terminal conditions equal to the corresponding No Protection Clean Prices Functions $\widehat{\Theta}$ at $\bar{T}$. So,

Theorem 4.9 (Hard Protection Clean Prices) In the case of $\bar{\tau}=\bar{T}$ for some $\bar{T} \leq T$, we define the following problems $(\mathcal{V I})$ on $\mathcal{D}=[0, \bar{T}] \times \mathbb{R}$ :

(a) Game Exchange Option

$$
\begin{gathered}
\min \left(-\mathcal{L} \bar{\Psi}+\mu \bar{\Psi}-\gamma\left(R^{c b}-R^{b}\right), \widehat{\Psi}-(\bar{P} \vee \kappa S-\widehat{\Phi})\right)=0, \quad t<\bar{T}, \\
\bar{\Psi}(\bar{T}, S)=\widehat{\Psi}(\bar{T}, S),
\end{gathered}
$$

(b) $R B$

$$
\begin{gathered}
\min \left(-\mathcal{L} \bar{\Pi}+\mu \bar{\Pi}-\left(\gamma R^{c b}+\Gamma\right), \bar{\Pi}-\bar{P} \vee \kappa S\right)=0, \quad t<\bar{T}, \\
\bar{\Pi}(\bar{T}, S)=\widehat{\Pi}(\bar{T}, S)
\end{gathered}
$$

Then for any of the problems (35) or (36), there exists a $\mathcal{P}$-solution on $\mathcal{D}$, denoted generically as $\bar{\Theta}(t, S)$, that determines the corresponding Hard Protection Clean Price, say $\bar{\Theta}_{t}$, in the sense that

$$
\bar{\Theta}_{t}=\bar{\Theta}\left(t, \widetilde{S}_{t}\right), \quad t \leq \bar{T} .
$$

Moreover, we have uniqueness of the $\mathcal{P}$-solution, and any stable, monotone and consistent approximation scheme for $\bar{\Theta}$ converges locally uniformly to $\bar{\Theta}$ on $\mathcal{D}$ as $h \rightarrow 0^{+}$.

Proof. For either problem, we know, by Theorem 4.7. that $\bar{\Theta}_{T}=\widehat{\Theta}\left(\bar{T}, \widetilde{S}_{T}\right)$ and, by Theorem 4.5 , that the terminal condition $\widehat{\Theta}(\bar{T}, \cdot)$ is continuous and in $\mathcal{P}$. Therefore, the result follows by an application of Theorems 4.3 , 3.3 (a) and 3.4(a).

Corollary 4.10 A Hard Protection Pre-default optimal stopping time $\tau_{p}^{*}$ for the Game Exchange Option problem, and for the RB problem as well, is given by

$$
\tau_{p}^{*}=\inf \left\{u \in[t, \bar{T}] ; \widetilde{S}_{u} \in \mathcal{E}_{h}\right\} \wedge T
$$

where

$$
\mathcal{E}_{h}=\left\{(t, S) \in[0, \bar{T}] ; \bar{\Pi}\left(u, \widetilde{S}_{t}\right)=\bar{P} \vee \kappa \widetilde{S}_{t}\right\} \wedge T
$$

is the Hard Protection Pre-default Put or Conversion Region.

Assuming that the RB is still alive at some time $t<\bar{T}$, we thus see that an optimal strategy for the holder of the RB consists in putting or converting, whichever is best, at the first hitting time of $\mathcal{E}_{h}$ (if any before $\tau_{d} \wedge \bar{T}$ ) by $S$. 


\subsubsection{Soft Call Protections}

Let us now treat the case of soft call protections. By an application of Theorems 4.3 3.3 (b) and 3.4(b), we have in turn the following result.

Theorem 4.11 (Soft Protection Clean Prices) Assuming that

$$
\bar{\tau}=\inf \left\{t>0 ; \widetilde{S}_{t} \geq \bar{S}\right\} \wedge T
$$

for some $\bar{S}>0$, we define the following problems $(\mathcal{V I})$ on $\mathcal{D}=[0, T] \times(-\infty, \bar{S}]$ :

(a) Game Exchange Option

$$
\begin{gathered}
\min \left(-\mathcal{L} \bar{\Psi}+\mu \bar{\Psi}-\gamma\left(R^{c b}-R^{b}\right), \bar{\Psi}-(\bar{P} \vee \kappa S-\widehat{\Phi})\right)=0, \quad t<T, S<\bar{S} \\
\bar{\Psi}(t, \bar{S})=\widehat{\Psi}(t, \bar{S}), \quad t \leq T \\
\bar{\Psi}(T, S)=(\kappa S-\bar{N})^{+}, \quad S \leq \bar{S}
\end{gathered}
$$

(b) $R B$

$$
\begin{gathered}
\min \left(-\mathcal{L} \bar{\Pi}+\mu \bar{\Pi}-\left(\gamma R^{c b}+\Gamma\right), \bar{\Pi}-\bar{P} \vee \kappa S\right)=0, \quad t<T, \\
\bar{\Pi}(t, \bar{S})=\widehat{\Pi}(t, \bar{S}), \quad t \leq T, \\
\bar{\Pi}(T, S)=\bar{N} \vee \kappa S, \quad S \leq \bar{S} .
\end{gathered}
$$

Then for any of the problems (37) or (38) there exists a $\mathcal{P}$-solution on $\mathcal{D}$, denoted generically as $\bar{\Theta}(t, S)$, that determines the corresponding Soft Protection Clean Price, say $\bar{\Theta}_{t}$, in the sense that

$$
\bar{\Theta}_{t}=\bar{\Theta}\left(t, \widetilde{S}_{t}\right), \quad t \leq \bar{\tau} .
$$

Moreover, we have uniqueness of the $\mathcal{P}$-solution, and any stable, monotone and consistent approximation scheme for $\bar{\Theta}$ converges locally uniformly to $\bar{\Theta}$ on $\mathcal{D}$ as $h \rightarrow 0^{+}$, provided it converges to $\bar{\Theta}(=\widehat{\Theta})$ at $\bar{S}$.

Corollary 4.12 $A$ Soft Protection Pre-default optimal stopping time $\tau_{p}^{*}$ for the Game Exchange Option problem, and for the RB problem as well, is given by

$$
\tau_{p}^{*}=\inf \left\{u \in[t, \bar{\tau}] ; \widetilde{S}_{u} \in \mathcal{E}_{s}\right\} \wedge T
$$

where

$$
\mathcal{E}_{s}=\{(t, S) \in[0, T] ; \bar{\Pi}(t, S)=\bar{P} \vee \kappa S\}
$$

is the Soft Protection Pre-default Put or Conversion Region.

So, assuming that the stock has not reached the level $\bar{S}$ yet, and that the RB is still alive, an optimal strategy for the holder of the RB consists in putting or converting, whichever is best, at the first hitting time of $\mathcal{E}_{s}$ (if any before $\tau_{d} \wedge \bar{\tau}$ ) by $\widetilde{S}$.

\subsection{Convertible Bonds with Positive Call Notice Period}

We now consider the case of a Convertible Bond with positive Call Notice Period.

Note that between the call time $t$ and the end of the notice period $t^{\delta}=(t+\delta) \wedge T$, a CB actually becomes a non-callable CS (denoted as PB, cf. Definition 3.2), that is, a CB with no call clause (formally, we set $\bar{\tau}=t^{\delta}$ in the related BSDE). For a fixed $t$, we call such a bond $t$-PB, and it has effective put payment equal to the effective call payment $C_{u}, u \in\left[t, t^{\delta}\right]$, of the original $\mathrm{CB}$, and effective payment at maturity $C_{t^{\delta}}$ (see [4]). 
Proposition 4.13 In the case of the $t-P B(t \in[0, T])$, the associated functions $f(u, S, \theta), g=g(S)$ and $\ell=\ell(u, S)$ are $(h=+\infty$ in all three cases below):

- $f(u, S, \theta)=\gamma(u, S) R^{b}(u, S)+\Gamma(u, S)-\mu(u, S) \theta, g(S)=\bar{C}, \ell(u, S)=-\infty$, for the embedded Bond (the $t$-Bond, in the sequel);

- $f(u, S, \theta)=\gamma(u, S)\left(R^{c b}-R^{b}\right)(u, S)-\mu(u, S) \theta, g(S)=\bar{C} \vee \kappa S-\widehat{\Phi}^{t}\left(t^{\delta}, S\right), \ell(u, S)=\bar{C} \vee \kappa S-$ $\widehat{\Phi}^{t}(u, S)$, where $\widehat{\Phi}^{t}$ is the No Protection Clean Price Function of the $t$-Bond (obtained by an application of Theorem 4.5, see also (39) below), for the embedded Game Exchange Option (the t-Game Exchange Option, in the sequel);

- $f(u, S, \theta)=\gamma(u, S) R^{c b}(u, S)+\Gamma(u, S)-\mu(u, S) \theta, g(S)=\bar{C} \vee \kappa S, \ell(u, S)=\bar{C} \vee \kappa S$, for the $t-P B$ itself.

Note that in view of the proof of Theorem 4.14(ii) below, it is convenient to define the related pricing problems on $\mathcal{D}^{t}:=\left[0, t^{\delta}\right] \times \mathbb{R}$, rather than merely on $\left[t, t^{\delta}\right] \times \mathbb{R}$.

Theorem 4.14 (Variational Inequalities for the embedded PBs) Given $t \in[0, T]$, we define the following problems $(\mathcal{V I})$ on $\mathcal{D}^{t}=\left[0, t^{\delta}\right] \times \mathbb{R}$ :

(a) $t$-Bond

$$
\begin{gathered}
-\mathcal{L} \widehat{\Phi}^{t}+\mu \widehat{\Phi}^{t}-\left(\gamma R^{b}+\Gamma\right)=0, \quad u<t^{\delta}, \\
\widehat{\Phi}^{t}\left(t^{\delta}, S\right)=\bar{C},
\end{gathered}
$$

(b) t-Game Exchange Option

$$
\begin{gathered}
\min \left(-\mathcal{L} \widehat{\Psi}^{t}+\mu \widehat{\Psi}^{t}-\gamma\left(R^{c b}-R^{b}\right), \widehat{\Psi}^{t}-\left(\bar{C} \vee \kappa S-\widehat{\Phi}^{t}\right)\right)=0, \quad u<t^{\delta}, \\
\widehat{\Psi}^{t}\left(t^{\delta}, S\right)=\bar{C} \vee \kappa S-\widehat{\Phi}^{t}\left(t^{\delta}, S\right),
\end{gathered}
$$

(c) $t-P B$

$$
\begin{gathered}
\min \left(-\mathcal{L} \widehat{\Pi}^{t}+\mu \widehat{\Pi}^{t}-\left(\gamma R^{c b}+\Gamma\right), \widehat{\Pi}^{t}-\bar{C} \vee \kappa S\right)=0, \quad u<t^{\delta}, \\
\widehat{\Pi}^{t}\left(t^{\delta}, S\right)=\bar{C} \vee \kappa S .
\end{gathered}
$$

(i) For any of the problems (VI) above, the corresponding Pre-default Clean t-Price $\widehat{\Theta}_{u}^{t}$ can be written as $\widehat{\Theta}^{t}\left(u, \widetilde{S}_{u}\right)$, where the function $\widehat{\Theta}^{t}$ is a $\mathcal{P}$-solution of $(\mathcal{V I})$ on $\mathcal{D}^{t}$. Moreover, we have uniqueness of the $\mathcal{P}$-solution, and any stable, monotone and consistent approximation scheme for $\widehat{\Theta}^{t}$ converges locally uniformly to $\widehat{\Theta}^{t}$ on $\mathcal{D}^{t}$ as $h \rightarrow 0^{+}$.

(ii) The function $\widehat{U}(t, S):=\widehat{\Pi}^{t}(t, S)$ is jointly continuous in time and space. Hence the function $\widetilde{U}(t, S)=\widehat{U}(t, S)+A_{t}$ is also continuous with respect to $(t, S)$, except for left jumps of size $-c^{i}$ at the $T_{i} s$.

Proof. Part (i) follows by an application of previous results, in view of Proposition 4.13 . We now prove (ii). Let $\left(t_{n}, S_{n}\right) \rightarrow(t, S)$ as $n \rightarrow \infty$. We decompose

$$
\widehat{\Pi}^{t_{n}}\left(t_{n}, S_{n}\right)=\widehat{\Pi}^{t}\left(t_{n}, S_{n}\right)+\left(\widehat{\Pi}^{t_{n}}\left(t_{n}, S_{n}\right)-\widehat{\Pi}^{t}\left(t_{n}, S_{n}\right)\right) .
$$

By (i), $\widehat{\Pi}^{t}\left(t_{n}, S_{n}\right) \rightarrow \widehat{\Pi}^{t}(t, S)$ as $n \rightarrow \infty$. Moreover, denoting $\widehat{C}_{t}=\bar{C} \vee \kappa \widetilde{S}_{t}, F=\gamma R^{c b}+\Gamma$, we have

$$
\alpha_{u} \widehat{\Pi}_{u}^{t}=\operatorname{esssup}_{\tau_{p} \in \mathcal{F}_{t^{\delta}}^{u}} \mathbb{E}_{\mathbb{Q}}\left(\int_{u}^{\tau_{p}} \alpha_{v} F_{v} d v+\alpha_{\tau_{p}} \widehat{C}_{\tau_{p}} \mid \mathcal{F}_{u}\right), \quad u \leq t^{\delta} .
$$

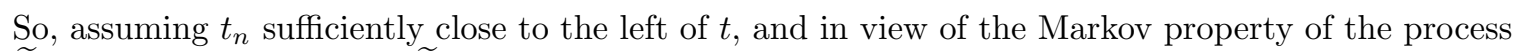
$\widetilde{S}$, we have, on the event $\left\{\widetilde{S}_{t_{n}}=S_{n}\right\}$,

$$
\begin{aligned}
& \alpha_{t_{n}} \widehat{\Pi}^{t_{n}}\left(t_{n}, S_{n}\right)=\operatorname{esssup}_{\tau_{p} \in \mathcal{F}_{t_{n}^{\delta}}^{t_{n}}} \mathbb{E}_{\mathbb{Q}}\left(\int_{t_{n}}^{\tau_{p}} \alpha_{v} F_{v} d v+\alpha_{\tau_{p}} \widehat{C}_{\tau_{p}} \mid \mathcal{F}_{t_{n}}\right) \\
& \leq \operatorname{esssup}_{\tau_{p} \in \mathcal{F}_{t^{\delta}}^{t_{n}}} \mathbb{E}_{\mathbb{Q}}\left(\int_{t_{n}}^{\tau_{p}} \alpha_{v} F_{v} d v+\alpha_{\tau_{p}} \widehat{C}_{\tau_{p}} \mid \mathcal{F}_{t_{n}}\right)=\alpha_{t_{n}} \widehat{\Pi}^{t}\left(t_{n}, S_{n}\right) .
\end{aligned}
$$


Conversely, for any $\tau_{p} \in \mathcal{F}_{t^{\delta}}^{t_{n}}$, we have $\tau_{p}{ }^{\prime}:=\tau_{p} \wedge t_{n}^{\delta} \in \mathcal{F}_{t_{n}^{\delta}}^{t_{n}}, 0 \leq \tau_{p}-\tau_{p}{ }^{\prime} \leq t-t_{n}$ and

$$
\begin{gathered}
\left|\int_{t_{n}}^{\tau_{p}} \alpha_{v} F_{v} d v+\alpha_{\tau_{p}} \widehat{C}_{\tau_{p}}-\int_{t_{n}}^{\tau_{p}{ }^{\prime}} \alpha_{v} F_{v} d v-\alpha_{\tau_{p}} \widehat{C}_{\tau_{p^{\prime}}}\right| \\
\quad \leq \int_{\tau_{p^{\prime}}}^{\tau_{p}} \alpha_{v}\left|F_{v}\right| d v+\left|\alpha_{\tau_{p}} \widehat{C}_{\tau_{p}}-\alpha_{\tau_{\tau^{\prime}}} \widehat{C}_{\tau_{p^{\prime}}}\right| .
\end{gathered}
$$

Therefore,

$$
\begin{aligned}
& \left|\mathbb{E}_{\mathbb{Q}}\left(\int_{t_{n}}^{\tau_{p}} \alpha_{v} F_{v} d v+\alpha_{\tau_{p}} \widehat{C}_{\tau_{p}} \mid \mathcal{F}_{t_{n}}\right)-\mathbb{E}_{\mathbb{Q}}\left(\int_{t_{n}}^{\tau_{p}{ }^{\prime}} \alpha_{v} F_{v} d v+\alpha_{\tau_{p^{\prime}}} \widehat{C}_{\tau_{\tau^{\prime}}} \mid \mathcal{F}_{t_{n}}\right)\right| \\
& \quad \leq \mathbb{E}_{\mathbb{Q}}\left(\int_{\tau_{\tau_{p}}{ }^{\prime}}^{\tau_{p}} \alpha_{v}\left|F_{v}\right| d v \mid \mathcal{F}_{t_{n}}\right)+\mathbb{E}_{\mathbb{Q}}\left(\left|\alpha_{\tau_{p}} \widehat{C}_{\tau_{p}}-\alpha_{\tau_{p^{\prime}}} \widehat{C}_{\tau_{p^{\prime}}}\right| \mid \mathcal{F}_{t_{n}}\right) \\
& \quad \leq a \sqrt{t-t_{n}}\|F\|_{\mathcal{H}^{2}}+\mathbb{E}_{\mathbb{Q}}\left(\left|\alpha_{\tau_{p}} \widehat{C}_{\tau_{p}}-\alpha_{\tau_{\tau^{\prime}}} \widehat{C}_{\tau_{p^{\prime}}}\right| \mid \mathcal{F}_{t_{n}}\right)
\end{aligned}
$$

for some finite, positive constant $a$. We conclude that $\widehat{\Pi}^{t_{n}}\left(t_{n}, S_{n}\right)-\widehat{\Pi}^{t}\left(t_{n}, S_{n}\right) \rightarrow 0$ as $t_{n} \rightarrow t^{-}$. But this is also true, with the same proof, as $t_{n} \rightarrow t^{+}$. Hence $\widehat{\Pi}^{t_{n}}\left(t_{n}, S_{n}\right)-\widehat{\Pi}^{t}\left(t_{n}, S_{n}\right) \rightarrow 0$ as $t_{n} \rightarrow t$. Finally, $\widehat{\Pi}^{t_{n}}\left(t_{n}, S_{n}\right) \rightarrow \widehat{\Pi}^{t}(t, S)$ as $t_{n} \rightarrow t$, as desired.

Theorem 4.15 A CB with positive notice period $\delta>0$ can be interpreted as an $R B$ with $\widetilde{U}^{c b}(t, S)=$ $\widetilde{U}(t, S)$, where $\widetilde{U}(t, S)$ is the function defined at Theorem 4.14(ii), so that (cf. 24))

$$
U_{t}^{c b}=\mathbb{1}_{\left\{\tau_{d}>t\right\}} \widetilde{U}\left(t, S_{t}\right)+\mathbb{1}_{\left\{\tau_{d} \leq t\right\}}\left(\bar{C} \vee \kappa S_{t}+A_{t}\right) .
$$

Proof. First, the $t$-PB related reflected BSDE has a solution, by Theorems 4.3 and 3.3 (a) applied to the $t$-PB. Thus the $t$-PB has a unique arbitrage price process $\Pi_{u}^{t}=\mathbb{1}_{\left\{u<\tau_{d}\right\}} \widetilde{\Pi}_{u}^{t}$ with $\Pi_{u}^{t}=\widehat{\Pi}_{u}^{t}+A_{u}$, by Theorem 3.2. Hence the arbitrage price of the CB upon call time $t$ (assuming the CB still alive at time $t$ ) is well defined, as $\Pi_{t}^{t}$, which is also equal to $\widetilde{U}\left(t, \widetilde{S}_{t}\right)$ (cf. Theorem 4.14(ii)).

Moreover, by Theorem 4.14(ii), the function $\widetilde{U}(t, S)$ is jointly continuous in time and space, except for negative left jumps of $-c^{i}$ at the $T_{i} \mathrm{~s}$ and $\Pi_{t}^{t} \geq \bar{C} \vee \kappa S_{t}+A_{t}$ on the event $\left\{\tau_{d}>t\right\}$, by the general results of [4]. Hence $U^{c b}$ defined as 42 satisfies all the requirements in (24).

Therefore, all the results of Section 4 are applicable to a CB, since the latter may be interpreted as an $R B$ in view of Theorem 4.15.

\section{$5 \quad$ Numerical Issues}

\subsection{Pricing}

Assume that $\bar{\tau}=0$ (no call protection) and that we have already specified all the parameters for one of the problems 32,33 or $(34)$, including, in the case of $(33)$ or $(34)$, the function $\widetilde{U}^{c b}$. Then one can solve the problem numerically (see e.g. 2, 29]) and it is known that, under mild conditions (cf. Theorem 3.4 and the Theorems of Section 4), suitable approximation schemes will converge towards the $\mathcal{P}$-solution of the problem as the discretization step goes to 0 . Solving the PDEs related to the embedded bond is standard, and therefore we shall not comment on this issue here.

To have a fully endogenous specification of the problem, one can take $\widetilde{U}^{c b}(t, S)=\widetilde{U}(t, S)$ as defined in Theorem 4.14(ii) in 33 or $(34)$, where $\widetilde{U}(t, S)$ is numerically computed by solving the related obstacle problems, using Theorem 4.14 (i). We provide below a practical algorithm for solving, say (34), with $\widetilde{U}^{c b}(t, S)=\widetilde{U}(t, S)$, using, for example, a fully implicit finite difference scheme (see, for instance, [33]) to discretize $\mathcal{L}$ : 
1. Localize problems (41) for the embedded $t$-PBs and problem (34) for the CB. A natural choice, for the t-PBs as for the $\mathrm{CB}$, is to localize the problems on the spatial domain $\left(-\infty, \frac{\bar{C}}{\kappa}\right]$, with a Dirichlet boundary condition equal to $\kappa S$ (or a Neumann boundary condition equal to $\kappa$ ) at level $\frac{\bar{C}}{\kappa}$;

2. Discretize the localized domain $\mathcal{D}^{l o c}=[0, T] \times\left(-\infty, \frac{\bar{C}}{\kappa}\right]$, using, say, one time step per day between 0 and $T$;

3. Discretize problems 41] for the embedded t-PBs on the subdomain $\left[t, t^{\delta}\right]$ of $\mathcal{D}^{l o c}$ for $t$ in the time grid (one problem per value of $t$ in the time grid);

4. Solve for $\widehat{\Pi}^{t}$ the discretized problems (41) corresponding to the embedded t-PBs for $t$ in the time grid (one problem per value of $t$ in the time grid);

5. Discretize problem (34) for the $\mathrm{CB}$ on $\mathcal{D}^{l o c}$ and solve the discretized problem, using the numerical approximation of $\widetilde{U}(t, S):=\widehat{\Pi}^{t}(t, S)+A_{t}$ as an input for $\widetilde{U}^{c b}(t, S)$ in 34 .

Since the problem for the $t$-PB only has to be solved on the time-strip $\left[t, t^{\delta}\right]$ of $\mathcal{D}^{l o c}$, the overall computational cost for solving a $\mathrm{CB}$ problem (34) with positive call notice period is roughly the same as that required for solving one $\mathrm{CB}$ problem without call notice period, plus the cost of solving $n$ PB problems that would be defined on the whole grid, where $n$ is the number of time mesh points in the notice period - that is typically one month, so $n=30$, for a notice period $\delta=1$ month and a time step of one day.

Finally if a call protection is in force then we proceed along essentially the same lines, using the results in Section 4.6 .

On Figure $1^{2}$ we plotted the price of the Convertible Bond, the embedded Bond and the embedded Game Exchange Option obtained in this way as a function of the stock level $S$ at time 0, in the simple case where $\delta=0$, no call protection is in force, and there are no dividends (no coupons nor recovery), and for the numerical data of remaining parameters gathered in Table 1 . We plotted in each case the curves corresponding to default intensities of the form $\gamma(t, S)=\gamma_{0}\left(\frac{S_{0}}{S}\right)^{\gamma_{1}}$ for $\gamma_{0}=0.02$ and $\gamma_{1}=1.2$ or $\gamma_{1}=0$, curves respectively labeled local and implied on each graph.

\begin{tabular}{|c|c|c|c|c|c|c|c|c|c|}
\hline$r$ & $q$ & $\eta$ & $\sigma$ & $S_{0}$ & $T$ & $\bar{P}$ & $\bar{N}$ & $\bar{C}$ & $\kappa$ \\
\hline $5 \%$ & 0 & 0 & $20 \%$ & 100 & $5 \mathrm{y}$ & 0 & 100 & 130 & 1 \\
\hline
\end{tabular}

Table 1: Parameter values

Note that in case $\alpha=1.2$, consistently with typical market data, the price of the CB as a function of $S$ exhibits the so-called ski-jump behavior, namely, it is convex for high values of $S$ and collapsing at the low values. This collapse at low levels of $S$ comes from the collapse of the embedded bond component of a CB ('collapse of the bond floor'). We refer the interested reader to [4 for more about this.

Remark 5.1 An alternative for pricing would be to use numerical methods for reflected BSDEs 32, 9, 10. Given the solution $(\Theta, Z, K)$ of a R2BSDE in a Markovian set-up, the interest of these methods is to provide numerical approximations not only to the state-process $\Theta$ (the price of the CS), but also to $Z$ (the 'delta' of the CS, cf. (21)). In our case, such methods would reduce to simple extensions to game problems of the well-known simulation methods for American options [30, 35, 31. Note however that these methods are not much used in the industry at this stage. Beyond the fact that they are computationally intensive, another reason is that they do not give a confidence interval, unlike standard Monte Carlo methods for European options. Yet, in order to take into account non standard soft call protection clauses, or, more generally, to cope with highly path-dependent features, it may be necessary to resort to such simulation methods.

\footnotetext{
${ }^{2}$ We thank Abdallah Rahal from the Mathematics Departments at University of Evry, France, and Lebanese University, Lebanon, for numerical implementation of the model and, in particular, for generating the picture.
} 

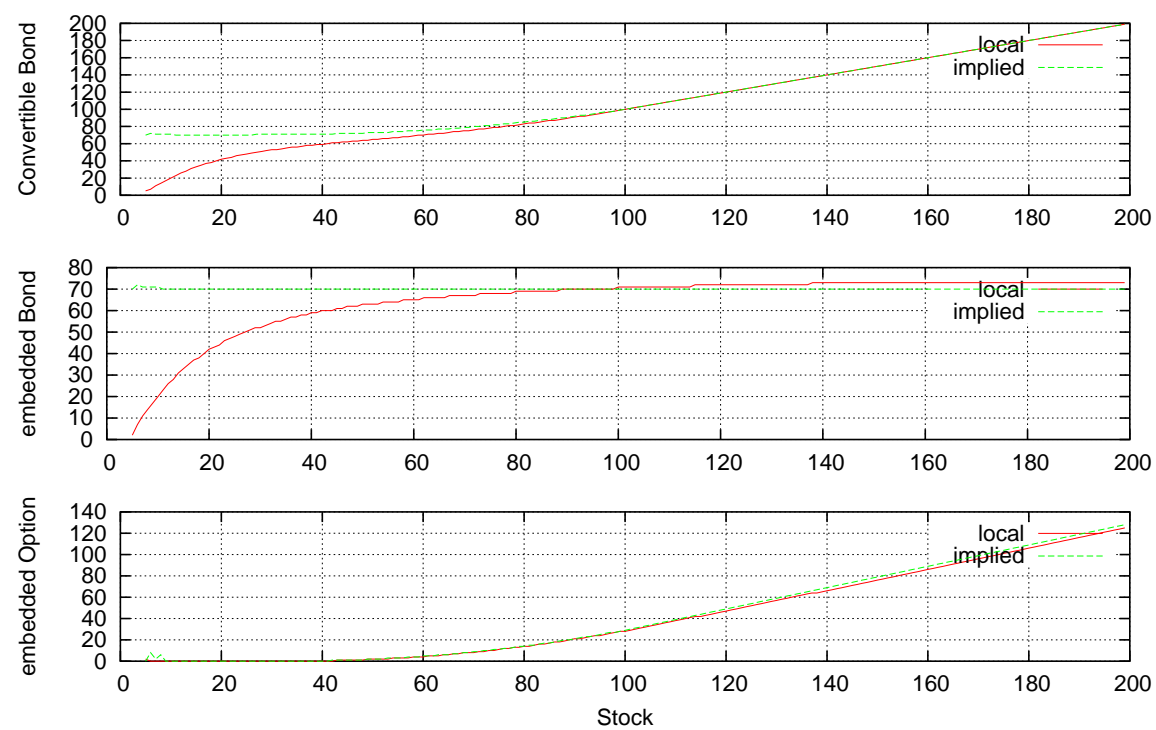

Figure 1: The Ski-Jump Diagram and its Decomposition

\subsection{Calibration}

A further numerical issue is the calibration of the model, which consists in fitting some specific parameters of the model, such as the local volatility $\sigma$ and the local intensity $\gamma$ in our model, to market prices of liquidly traded assets. Various input instruments can be used in this calibration process, such as: vanilla options on the underlying equity and/or CDS traded on bonds of the issuer (see, e.g., [1]).

As it can be seen on Figure 1, the price of the embedded game exchange option enjoys much better properties than the price of the $\mathrm{CB}$ in terms of convexity with respect to the stock price, and thus in turn (see [4]), in terms of monotonicity with respect to the volatility. These simple numerical experiments also support the intuitive guess that the embedded bond concentrates most of the interest rate and credit risks of a convertible bond, whereas the embedded game exchange option concentrates most of the volatility risk (note in this respect that the embedded game exchange option always has a coupon process equal to zero). These features suggest that it could be advantageous to use prices of (synthetic) embedded game exchange options, rather than prices of CBs, for the purpose of calibration.

We refer the reader to the last section of [4] for a more complete discussion of potential benefits of our decomposition of a convertible bond into bond and option components for calibration purposes.

\section{A Rolling CDS}

In this section we derive the dynamics of the rolling CDS of Section 2.2.1, in the context of the Markovian defaultable diffusion model of this paper. We refer the interested reader to [7] for the 
dynamics of a rolling CDS in a more general set-up. Since the derivation takes a much simpler form in the present Markovian situation, we provide a direct and self-contained proof.

It was shown in [7] that the cumulative price process $\widehat{B}$ of a rolling CDS satisfies (using the set-up of present paper)

$$
d\left(\beta_{t} \widehat{B}_{t}\right)=\left(1-H_{t}\right) \beta_{t} \alpha_{t}^{-1}\left(d p_{t}-\bar{\nu}\left(t, \widetilde{S}_{t}\right) d f_{t}\right)+\beta_{t} \nu(t) d M_{t}^{d},
$$

where $d p$ and $d f$ denote the stochastic differentials of the following processes, with fixed value $\theta=\bar{T}(t)$ of the $\theta$-parameter therein (that is, stochastic differentials with respect to $t$ in $\mathcal{F}_{t}$ only, not in $\theta=\bar{T}(t))$ :

$$
p_{t}=\mathbb{E}_{\mathbb{Q}}\left(\int_{0}^{\theta} \nu(u) \alpha_{u} \gamma\left(u, \widetilde{S}_{u}\right) d u \mid \mathcal{F}_{t}\right)
$$

and

$$
f_{t}=\mathbb{E}_{\mathbb{Q}}\left(\int_{0}^{\theta} \alpha_{u} d u \mid \mathcal{F}_{t}\right)
$$

It is rather straightforward to verify that the (local) martingale $P$ given as

$$
P_{t}=\int_{0}^{t} \alpha_{u}^{-1} d p_{u}
$$

is equal to the martingale part of the process $\widehat{p}$ defined as

$$
\widehat{p}_{t}=\mathbb{E}_{\mathbb{Q}}\left(\int_{t}^{\theta} \nu(u) \alpha_{t}^{-1} \alpha_{u} \gamma\left(u, \widetilde{S}_{u}\right) d u \mid \mathcal{F}_{t}\right) .
$$

In particular, in view of our Markovian set-up, process $P_{t}$ can also be written as

$$
P_{t}=\int_{0}^{t} \partial_{S} \widetilde{P}\left(u, \widetilde{S}_{u}\right) \sigma\left(u, \widetilde{S}_{u}\right) \widetilde{S}_{u} d W_{u}
$$

where the function $\widetilde{P}$ is the pre-default pricing function of a protection rate payment $\nu(u)$ with horizon $\theta$.

Likewise, it is straightforward to verify that the (local) martingale $F$ given as

$$
F_{t}=\int_{0}^{t} \alpha_{u}^{-1} d f_{u}
$$

is equal to the martingale part of the process $\widehat{f}$ defined as

$$
\widehat{f_{t}}=\mathbb{E}_{\mathbb{Q}}\left(\int_{t}^{\theta} \alpha_{t}^{-1} \alpha_{u} d u \mid \mathcal{F}_{t}\right) .
$$

Thus, process $F_{t}$ can also be written as

$$
F_{t}=\int_{0}^{t} \partial_{S} \widetilde{F}\left(u, \widetilde{S}_{u}\right) \sigma\left(u, \widetilde{S}_{u}\right) \widetilde{S}_{u} d W_{u}
$$

where the function $\widetilde{F}$ is the pre-default pricing function of a unit rate fee payment with horizon $\theta$. This demonstrates validity of $(7)$. 


\section{B Viscosity Solutions of Double Obstacle Variational Inequal- ities}

We give in this section the definition of viscosity solutions which is required, in the case of our obstacles problem $(\mathcal{V I})$, to cope in particular with the potential discontinuities in time of $f$ at the $T_{i}$ s (cf. 18p). We refer the interested reader to Crépey [14] for every detail on this issue. We set, for $i=1,2, \ldots, K(\mathrm{cf}$. 22, $)$,

$$
\mathcal{D}^{i}=\mathcal{D} \cap\left\{T_{i-1} \leq t \leq T_{i}\right\}, \operatorname{Int}_{p} \mathcal{D}^{i}=\operatorname{Int}_{p} \mathcal{D} \cap\left\{T_{i-1} \leq t<T_{i}\right\}, \quad \partial_{p} \mathcal{D}^{i}=\mathcal{D}^{i} \backslash \operatorname{Int}_{p} \mathcal{D}^{i} .
$$

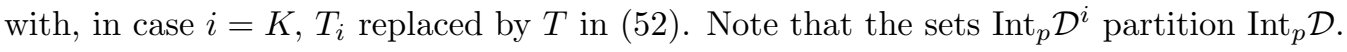

Definition B.1 (i) A locally bounded upper semicontinuous function $\Theta$ on $\mathcal{D}$ is called a viscosity subsolution of $(\mathcal{V I})$ on $\operatorname{Int}_{p} \mathcal{D}$ if and only if $\Theta \leq h$, and $\Theta(t, S)>\ell(t, S)$ implies

$$
-\mathcal{L} \varphi(t, S)-f(t, S, \Theta(t, S)) \leq 0
$$

for any $(t, S) \in \operatorname{Int}_{p} \mathcal{D}^{i}$ and $\varphi \in \mathcal{C}^{1,2}\left(\mathcal{D}^{i}\right)$ such that $\Theta-\varphi$ is maximal on $\mathcal{D}^{i}$ at $(t, S)$, for some $i \in 1,2, \ldots, K$.

(ii) A locally bounded lower semicontinuous function $\Theta$ on $\mathcal{D}$ is called a viscosity supersolution of $(\mathcal{V I})$ on $\operatorname{Int}_{p} \mathcal{D}$ if and only if $\Theta \geq \ell$, and $\Theta(t, S)<h(t, S)$ implies

$$
-\mathcal{L} \varphi(t, S)-f(t, S, \Theta(t, S)) \geq 0
$$

for any $(t, S) \in \operatorname{Int}_{p} \mathcal{D}^{i}$ and $\varphi \in \mathcal{C}^{1,2}\left(\mathcal{D}^{i}\right)$ such that $\Theta-\varphi$ is minimal on $\mathcal{D}^{i}$ at $(t, S)$, for some $i \in 1,2, \ldots, K$.

(iii) $\Theta$ is called a viscosity solution of $(\mathcal{V I})$ on $\operatorname{Int}_{p} \mathcal{D}$ if and only if it is both a viscosity subsolution and a viscosity supersolution of $(\mathcal{V I})$ on $\operatorname{Int}_{p} \mathcal{D}$ - in which case, $\Theta$ is a continuous function.

Remark B.2 (i) In case of a CS with no discrete coupons (like for instance the Game Exchange Option component of a CB, which is a zero-coupon CS), the previous definitions reduce to the standard definitions of viscosity (semi-)solutions for obstacles problems (see, for instance, [23, 12]). (ii) A classical solution of $(\mathcal{V} \mathcal{I})$ on $\operatorname{Int}_{p} \mathcal{D}$ (if any) is necessarily a viscosity solution of $(\mathcal{V} \mathcal{I})$ on $\operatorname{Int}_{p} \mathcal{D}$.

(iii) A viscosity subsolution (resp. supersolution) $\Theta$ of $(\mathcal{V} \mathcal{I})$ on $\operatorname{Int}_{p} \mathcal{D}$ does not need to verify $\Theta \geq \ell$ (resp. $\Theta \leq h$ ) on $\operatorname{Int}_{p} \mathcal{D}$. A viscosity solution (in particular, a classical solution, if any) $\Theta$ of $(\mathcal{V} \mathcal{I}$ ) on $\operatorname{Int}_{p} \mathcal{D}$ necessarily satisfies $\ell \leq \Theta \leq h$ on $\operatorname{Int}_{p} \mathcal{D}$.

\section{References}

[1] Andersen, L. And Buffum, D.: Calibration and implementation of convertible bond models. Working paper, Bank of America Securities, 2003.

[2] Ayache, E., Forsyth, P. and Vetzal, K.: Valuation of convertible bonds with credit risk. The Journal of Derivatives, Fall 2003.

[3] Barles, G. And Souganidis, P.E.: Convergence of approximation schemes for fully nonlinear second order equations. Asymptotic Anal. 4 (1991), 271-283.

[4] Bielecki, T.R., Crépey, S., Jeanblanc, M. and Rutkowski, M.: Arbitrage pricing of defaultable game options with applications to convertible securities. Forthcoming in Quantitative Finance.

[5] Bielecki, T.R., Crépey, S., Jeanblanc, M. and Rutkowski, M.: Valuation and hedging of defaultable game options in a hazard process model. Working Paper available online at www.defaultrisk. com, 2007. 
[6] Bielecki, T.R., Crépey, S., Jeanblanc, M. And Rutkowski, M.: Defaultable options in a Markovian intensity model of credit risk. Mathematical Finance, Forthcoming (New Updated Version available online at ww. defaultrisk.com).

[7] Bielecki, T.R., Jeanblanc, M. and Rutkowski, M.: Pricing and trading credit default swaps. Work in preparation.

[8] Bielecki, T.R. And Rutkowski, M.: Credit Risk: Modeling, Valuation and Hedging. Springer-Verlag, Berlin, 2002.

[9] Bouchard, B. And Chassagneux, J.-F.: Discrete time approximation for continuously and discretely reflected BSDE's. Preprint, 2006.

[10] Bouchard, B. And Touzi, N.: Discrete-time approximation and Monte Carlo simulation of backward stochastic differential equations. Stochastic Processes and their Applications 111 (2004), 175-206.

[11] Carr, P. And Linetsky, V.: A jump to default extended CEV model: an application of Bessel processes. Finance and Stochastics 10 (2006), 303-330.

[12] Crandall, M., Ishit, H., And Lions, P.-L.: User's guide to viscosity solutions of second order partial differential equations. Bulletin of the American Mathematical Society 27 (1992), $1-67$.

[13] CrÉpey, S.: Markovian reflected and doubly reflected BSDEs in a jump-diffusion setting with regimes. Submitted.

[14] CRÉPEy, S.: About the pricing equation in finance. Submitted.

[15] Crépey, S., Matoussi, A.: About the Greeking equation in finance. Work in preparation.

[16] Crépey, S., Matoussi, A.: Reflected and doubly reflected BSDEs with jumps: A priori estimates and comparison principle. Submitted.

[17] Darling, R. And PARdoux, E.: Backward SDE with random terminal time and applications to semilinear elliptic PDE. Annals of Probability 25 (1997), 1135-1159.

[18] Davis, M. And LischkA, F.R.: Convertible bonds with market risk and credit risk. In: Applied Probability, Studies in Advanced Mathematics 26, Edited by Chan R., Kwok Y.K., Yao D. and Zhang Q., American Mathematical Society and International Press, 2002, pp. $45-58$.

[19] Duffie, D. And Singleton, K.J.: Modeling term structures of defaultable bonds. Review of Financial Studies 12 (1999), 687-720.

[20] El Karoui, N., Hamadène, S. and Matoussi, A.: Backward stochastic differential equations and applications. Forthcoming in: Paris-Princeton Lecture Notes on Mathematical Finance, Edited by Carmona, R. et al., Springer-Verlag, Berlin.

[21] El Karoui, N., Kapoudjian, E., Pardoux, C., Peng, S., and Quenez, M.-C.: Reflected solutions of backward SDEs, and related obstacle problems for PDEs. Annals of Probability 25 (1997), 702-737.

[22] El Karoui, N., Peng, S., And Quenez, M.-C.: Backward stochastic differential equations in finance. Mathematical Finance 7 (1997), 1-71.

[23] Fleming, W. And Soner, H.: Controlled Markov Processes and Viscosity Solutions, Second Edition. Springer-Verlag, Berlin, 2006.

[24] Jacod, J. And Shiryaev, A.N.: Limit Theorems for Stochastic Processes. Springer-Verlag, 2003. 
[25] Kallsen, J. And Kühn, C.: Convertible bonds: financial derivatives of game type. In: Exotic Option Pricing and Advanced Lévy Models, Edited by Kyprianou, A., Schoutens, W. and Wilmott, P., Wiley, 2005, pp. 277-288.

[26] Kifer, Y.: Game options. Finance and Stochastics 4 (2000), 443-463.

[27] KusuokA, S.: A remark on default risk models. Advances in Mathematical Economics 1 (1999), 69-82.

[28] Linetsky, V.: Pricing equity derivatives subject to bankruptcy. Mathematical Finance 16 (2006), 255-282.

[29] Kwok, Y. And LaU, K.: Anatomy of option features in convertible bonds. Journal of Futures Markets 24 (2004), 513-532.

[30] Longstaff, F.A. And Schwartz, E.S.: Valuing American options by simulations: a simple least-squares approach. Review of Financial Studies 14 (2001), 113-147.

[31] Lvov, D., Yigitbasioglu, A.B. And El Bachir, N.: Pricing convertible bonds by simulation. Working paper, 2004.

[32] Ma, J. AND Zhang, J.: Representations and regularities for solutions to BSDEs with reflections. Stochastic Processes and their Applications 115 (2005), 539-569.

[33] Morton, K. And Mayers, D.: Numerical Solution of Partial Differential Equations. Cambridge University Press, Cambridge, 1994.

[34] Pardoux, E. and Peng, S.: Backward stochastic differential equations and quasilinear parabolic partial differential equations, In: Stochastic Partial Differential Equations and Their Applications. Lecture Notes in Control Inf. Sci. 176, Springer-Verlag, Berlin, 1992, pp. 200217.

[35] Tsitsiklis, J.N. And Van Roy, B.: Regression methods for pricing complex American-style options. IEEE Transactions on Neural Networks 12 (2001), 694-703. 